\title{
Una revisión sistemática de literatura sobre la violencia contra mujeres mayores en América Latina y el Caribe: ¿se ha alcanzado una perspectiva interseccional?
}

\section{Gabriela Ramos Bonilla}

(iD) https://orcid.org/0000-0002-0786-1028

Pontificia Universidad Católica del Perú ramos.g@pucp.pe

RESUMEN

Este artículo hace una revisión crítica y sistemática de 72 investigaciones cualitativas y cuantitativas relacionadas con la violencia ejercida contra mujeres mayores de América Latina y el Caribe (ALC) entre los años 2000 y 2020 con el objetivo de evaluar en qué medida han logrado analizar este fenómeno desde un enfoque interseccional - vinculado con el género y la edad en simultáneo- y cuáles son sus brechas, limitaciones y principales hallazgos. Esta investigación demuestra que el estudio sobre este tema ha sido dominado por los marcos conceptuales del «maltrato a las personas adultas mayores»y la «violencia contra la mujer», así como también por una perspectiva médica y cuantitativa que no permiten comprender este problema cabalmente, pues abordan la violencia de manera fragmentada.

En comparación, un grupo novedoso de estudios sociales utilizó un marco conceptual interseccional abordando la violencia desde un enfoque de curso vital y una perspectiva fenomenológica. A modo de conclusión se plantea que los estudios interseccionales, a pesar de ser aún escasos en ALC, poseen un gran potencial para la comprensión de la naturaleza acumulativa y la interconexión entre diferentes experiencias de abuso, asi como explorar los sistemas de creencias de mujeres mayores, sus experiencias cotidianas de abuso, y sus dilemas, barreras y estrategias para buscar ayuda. Esta perspectiva permitirá 
la construcción de teoría adaptada a nuestros contextos culturales de forma inductiva y basada en evidencia contextualizada es un arma poderosa para enfrentar falencias de los marcos teóricos anteriores.

Palabras clave: mujer adulta mayor, violencia contra la mujer, maltrato a las personas adultas mayores, interseccionalidad, América Latina y el Caribe.

\section{A critical literature review on violence against older women in Latin America and the Caribbean: has an intersectional research approach been achieved?}

ABSTRACT

This article makes a critical and systematic review of 72 qualitative and quantitative studies related to the subject of violence against older women (VOW) in LAC between the years 2000 and 2020. It aims to evaluate to what extent they have managed to analyse this phenomenon using an intersectional approach - as a gender - and age-based problem simultaneously and the gaps, limitations and main findings of this body of research. This review shows that this subject's study has been dominated by the conceptual frameworks of «elder abuse» and «violence against women» (VAW). They approached this subject through a medical perspective and quantitative methodologies and failed to fully understand it since they addressed violence in a fragmented way. In comparison, a novel group of studies carried out by social researchers through the conceptual framework of «intersectionality" approached violence from a life-course and phenomenological perspective. This research concludes that even though intersectional studies are still scarce in $L A C$, they show great potential to reveal the cumulative nature of violence, the interconnection between different lifetime experiences of abuse, to explore older women's belief systems, their daily experiences of abuse, dilemmas, barriers and strategies for seeking help. This framework will allow the construction of theory inductively and grounded on contextualised evidence is a powerful weapon to confront former theoretical frameworks.

Keywords: older women, violence against women, elder abuse, intersectionality, Latin America and the Caribbean. 


\section{INTRODUCCIÓN}

Las mujeres mayores en Latinoamérica y el Caribe (ALC) están aumentando a uno de los ritmos más rápidos del mundo frente a otros grupos etarios. Esto debería situarlas en el centro de discusiones académicas y de política pública en la región. Desde la mitad del siglo pasado, la población de ALC ha experimentado una dramática transformación en su estructura de edad debido al envejecimiento poblacional ${ }^{1}$. Los procesos demográficos han generado un aumento en la esperanza de vida a la edad de sesenta años de las mujeres mayores, que se ha incrementado de 15,8 a 23,4 años extra entre 1950 y 2020. Como consecuencia, el número y proporción de mujeres mayores ha aumentado de manera dramática entre 1950 y 2020 del 6\% (5 millones) al 14\% (46 millones) del total de la población femenina, y seguirá aumentando hasta representar el 27\% (104 millones) en el año 2050 (Leeson, 2013, p. 62; BID, 2015, p. 44; Huenchuan, 2018, p. 24; Naciones Unidas, 2020, p. 11; Naciones Unidas, 2019).

Envejecer es una experiencia heterogénea para las mujeres debido a que ALC es considerada la región más desigual del mundo (CEPAL, 2016, p. 14). Si bien un grupo privilegiado de mujeres mayores es capaz de envejecer con bienestar, la mayoría enfrenta un gran número de dificultades en la vejez producto de las desigualdades sociales acumuladas a lo largo de sus vidas. Como grupo, las mujeres mayores presentan necesidades diferentes que los hombres mayores, debido a que muchas de ellas no solo están envejeciendo en contextos de pobreza y desigualdad estructural, sino que también están sufriendo los resultados de

\footnotetext{
La reducción de las tasas de fertilidad de 5,5 a 2,1 hijos por mujer en los períodos de 1965 1970 a 2015-2020 y la disminución de la mortalidad infantil (1960-2020) de 101 a 13 muertes por cada 1000 nacidos vivos permitieron el aumento de la esperanza de vida al nacer, de 59 años en 1960 a 76 hasta la fecha en ALC. Así, la región ha cambiado completamente, desde tener una población joven con una pequeña proporción de adultos mayores - alrededor del $6 \%$ - hasta experimentar un acelerado proceso de envejecimiento en la actualidad. Se prevé que el porcentaje de adultos mayores aumentará del 13\% al 25\% entre los años 2020 y 2050 (Saad, 2011, p. 55; Leeson, 2013, p. 65; Naciones Unidas, 2019).
} 
la desigualdad de género. La división del poder y el trabajo en las sociedades de ALC privilegia a los hombres y desfavorece a las mujeres, excluyéndolas de la toma de decisiones y estableciendo barreras de género en el acceso a los servicios educativos y al mercado laboral debido a su rol de cuidadoras familiares no remuneradas (Markides et al., 2010, p. 160; Daichman y Giraldo, 2013, p. 134; Huenchuan, 2018, p. 12; Manso y Lopes, 2020). Por esta razón, las mujeres en ALC muestran menor tasa de participación laboral que los hombres (56\% comparado con $83 \%$ ) y presentan mayores tasas de pobreza, lo cual tiene relación con el hecho de que solo el $52 \%$ de mujeres mayores cuenta con una pensión de jubilación, comparado con el 62,3\% de hombres mayores. Este fenómeno social, que ha sido llamado la «feminización de la pobreza", afecta el acceso de mujeres adultas mayores a una adecuada nutrición, vivienda, salud, actividades sociales y otras necesidades básicas (Kalache et al., 2005: 38; Giraldo, 2010, p. 154; Cotlear y Tornarolli, 2011, p. 108; Dulcey-Ruiz, 2013, p. 258; IDB, 2015, p. 18; Huenchuan, 2018, p. 62).

Adicionalmente, el 49\% de mujeres mayores en ALC presentan múltiples enfermedades crónicas que limitan su funcionalidad, un porcentaje mayor que el de sus contrapartes masculinas (Huenchuan, 2018, p. 74). Esta diferencia también se origina en las desigualdades de género, en tanto el deterioro de la salud es producto de la falta de acceso a servicios médicos, la mala nutrición y los múltiples embarazos, partos y abortos a lo largo del curso vital (Arilha et al., 2003, p. 10; Manso y Lopes, 2020, p. 63). Por esta razón, a pesar de que las mujeres viven más, presentan más años de vida enfermas. La falta de sistemas formales de seguridad en la región lleva a las mujeres mayores a depender de sus familiares más jóvenes para recibir atención y apoyo informal. Sin embargo, este tipo de apoyo se está debilitando debido a las tendencias hacia menores tasas de fertilidad y aumento de la migración en la región (Estes, 2005, p. 555; Kalache et al., 2005, p. 37; Kinsella, 2009, pp. 24-25; Dulcey-Ruiz, 2013, p. 258).

Las condiciones de vulnerabilidad en las que muchas mujeres mayores de ALC viven las pone en mayor riesgo de experimentar discriminación y violencia. Sin embargo, poco se sabe acerca de esta problemática, debido a que el interés académico y político sobre este tema es relativamente reciente en la región (Calsanti, 2010, p. 140; Motta, 2010; Daichman y Giraldo, 2013, pp. 136-138; Manso y Lopes, 2020, p. 73). Esa brecha de conocimiento se debe a la falta de datos poblacionales representativos sobre el tema. Por un lado, las encuestas sobre violencia contra las mujeres han permitido a los gobiernos reconocer que la violencia de género es generalizada en ALC y experimentada por el 30\% de 
las mujeres que alguna vez han tenido pareja (OMS, 2013, p. 16). Sin embargo, estos datos - usualmente recogidos a través de las Encuestas de Demografía y de Salud (DHS) o de Salud Reproductiva (RHS) - excluyen a las mujeres de mediana edad y adultas mayores de sus muestras: se centran en las mujeres en edad reproductiva de 15 a 49 años $^{2}$ (Bott et al., 2012, p. xv; HelpAge International, 2017a, p. 33).

Por otro lado, las encuestas sobre maltrato a personas mayores en la región son escasas y solo se han realizado en México, Ecuador y Colombia, lo que aún no permite brindar un panorama regional (Daichman y Giraldo, 2013, p. 134; OMS, 2014, p. 15). Debido a la falta de datos poblacionales, la única información disponible son los registros administrativos generados por los servicios de protección locales, que dan cuenta de las denuncias de violencia realizadas por mujeres mayores. Esta brecha de información sobre la violencia ejercida en contra de las mujeres mayores en países en desarrollo ha ocasionado que solo se tenga un panorama parcial de la prevalencia y la naturaleza de esta problemática en ALC. Ello ha llevado al desarrollo de políticas, servicios y teorías basadas en información incompleta, sin tomar en cuenta las experiencias y necesidades de una población creciente de mujeres mayores en la región (OMS e INPEA, 2002, p. 4; Fisher et al., 2003, p. 1412; Zink et al., 2003, p. 1440; Naciones Unidas, 2013, p. 16; Cheung et al., 2015, p. 429; Crockett y Cooper, 2016, p. 6; HelpAge International, 2017b, p. 3; Bows, 2019, p. 4; Meyer et al., 2019, p. 2).

Debido a la falta de datos representativos nacionales sobre violencia contra las mujeres mayores en ALC, las principales fuentes de evidencia en la materia son un grupo de estudios académicos que han emergido durante las últimas décadas, pero que no habían sido suficientemente sistematizados y evaluados hasta la fecha (Daichman y Giraldo, 2013, p. 143). En ese sentido, la presente investigación hace una revisión crítica y sistemática de esta literatura con el objetivo de evaluar en qué medida ha logrado analizar este fenómeno desde un enfoque interseccional — relacionado con el género y la edad en simultáneo-y cuáles son las brechas de conocimiento aún existentes, limitaciones y principales hallazgos o contribuciones.

2 Solo cuatro países de la región (Ecuador, México, Uruguay y Perú) han creado recientemente encuestas nacionales centradas en la VCM sin límite de edad. Sin embargo, estos datos todavía apenas han sido analizados para explorar las experiencias de violencia contra las mujeres adultas mayores (Cepal, 2014, pp. 34-36). 


\section{MARCO TEÓRICO EN EL ESTUDIO DE VIOLENCIA CONTRA MUJERES MAYORES}

El estudio de la violencia contra las mujeres mayores ha sido dominado por los marcos conceptuales y teóricos como «violencia contra la mujer» (en adelante VCM) y «maltrato y abuso a la persona adulta mayor» (en adelante MPAM). Sin embargo, el interés en las mujeres mayores y sus experiencias de violencia es aún reciente —empezó en las décadas de 1980 y 1990 — debido a que ambos campos de estudio han demostrado poco interés en esta temática. Asimismo, el marco conceptual de la interseccionalidad será presentado para discutir las posibilidades que brinda para el entendimiento de este fenómeno como una problemática asociada a la edad y el género de manera simultánea. Este debate será usado para evaluar en qué medida la literatura académica latinoamericana ha logrado conectar las desigualdades basadas en género y edad en el estudio de la violencia contra mujeres mayores de la región.

\section{Violencia contra la mujer y el maltrato y abuso a la persona adulta mayor}

El estudio de la VCM nació durante las décadas de 1960 y 1970, con el movimiento de mujeres maltratadas - battered women en inglés - en los Estados Unidos. Este movimiento de intelectuales y activistas feministas tuvo como objetivo evidenciar públicamente la existencia de la VCM e identificar gradualmente este problema como un asunto de interés público y un crimen sobre el cual los Estados tienen la obligación de intervenir. Las investigaciones y acciones impulsadas por este movimiento se basaban en las teorías del cambio social buscando la transformación de las relaciones de género, así como también empoderar a la víctima. Por ello, consideraban que la escucha de las voces de las sobrevivientes era un elemento fundamental (Band-Winterstein y Eisikovits, 2010, p. 203; Hightower, 2010, pp. 19-20; Straka y Montminy, 2006, p. 262).

Contar con un concepto consensuado de VCM fue un proceso complicado. Finalmente, en 1996 los Centros para el Control y la Prevención de Enfermedades de Estados Unidos acuñaron el concepto de «violencia íntima de pareja», el cual ha guiado la investigación y la recopilación de datos desde entonces. Esta definición incluyó distintos tipos de violencia - física, sexual y psicológica» y se identificó como principales perpetradores a los cónyuges, parejas y extraños (Bonomi et al., 2007, pp. 34-35; Hightower, 2010, pp. 18-20; Naciones Unidas, 2013, pp. 7-9; Crockett et al., 2015, p. 293). 
A pesar de que los investigadores de VCM enfatizaron que este problema afectaba a un grupo grande y diverso de mujeres de todas las edades, dejaron de lado las experiencias de violencia de las mujeres mayores, centrándose en el trabajo con mujeres en edad reproductiva (Vinton, 1991, pp. 5-6; Fisher et al., 2003, p. 1409; Hightower, 2004, p. 61; Calasanti y Slevin, 2006, p. 2; Crockett et al., 2015, p. 294; Straka y Montminy, 2006, p. 262). Debido a ello, Hightower (2010, p. 21) interpela al movimiento feminista y pregunta provocativamente si esta falta de atención a las mujeres mayores no es una demostración de la discriminación por edad dentro de la academia.

En comparación, la investigación e intervención relacionadas con el MPAM comenzó a fines de la década de 1970 y principios de 1980 como producto de la preocupación de gerontólogos sociales y profesionales de la salud. Estos primeros investigadores y activistas tenían como objetivo generar mejores mecanismos de atención de las necesidades de personas mayores víctimas de violencia. Este grupo consideraba que la vejez era el principal factor de riesgo ante la violencia doméstica y consideraban que la principal causa de este problema era la sobrecarga o estrés que sentían los cuidadores por tener que atender a una persona mayor dependiente (Meyer et al., 2019: 2; Straka y Montminy, 2006, p. 262).

El campo de estudios sobre MPAM también tuvo dificultades para definir de manera consensuada lo que se entendería por «maltrato y abuso a las personas mayores». Por un lado, el concepto de «maltrato a las personas adultas mayores» fue creado por Action on Elder Abuse (1995), una organización no gubernamental británica, siguiendo un enfoque de gerontología social. Posteriormente, fue adoptado y popularizado por la Red Internacional para la Prevención del Abuso y Maltrato en la Vejez (INPEA, 1997) y la Organización Mundial de la Salud (OMS) en 2002. Esta definición establecía que «el maltrato a las personas adultas mayores se define como una acción única o repetida, o la falta de la respuesta apropiada que ocurre dentro de cualquier relación donde exista una expectativa de confianza y la cual produzca daño o angustia a una persona anciana» (OMS e INPEA, 2002).

Por otro lado, el concepto de «abuso de adultos mayores vulnerables» fue acuñado por un panel de expertos del Consejo Nacional de Investigación de la Academia Nacional de Ciencias de los Estados Unidos ${ }^{3}$ en el año 2003, siguiendo una perspectiva geriátrica o médica enfocada en la violencia perpetrada por los cuidadores de ancianos dependientes en entornos domésticos e institucionales. Por

\footnotetext{
Fue creado por el «Expert Panel to Review Risk and Prevalence of Elder Abuse and Neglect».
} 
ello, se definió este tipo de abuso como «acciones intencionales que causen daño o creen un riesgo grave de daño (sea o no intencional) a una persona mayor vulnerable por parte de un cuidador u otra persona con la que mantenga una relación de confianza, o la falla de un cuidador por satisfacer las necesidades básicas o la protección de la persona mayor ante algún daño» (Naciones Unidas, 2013, p. 4). Ambos conceptos contemplan un amplio rango de tipos de violencia - física, psicológica o emocional, financiera, sexual y negligencia - y de perpetradores como la pareja, otros familiares como los hijos adultos y nietos, así como no familiares, como los extraños y cuidadores.

El movimiento MPAM ha recibido tres críticas importantes. Primero, Brandl (2006, p. 40) señaló que parece culpar a la víctima porque considera que la causa de este problema es la dependencia de la persona mayor y la sobrecarga del cuidado. La segunda crítica fue que este movimiento no suele contemplar la participación activa de las víctimas, lo cual está vinculado a la asociación de la vejez con un estado de dependencia y falta de agencia. La tercera crítica fue que el análisis del MPAM no toma en cuenta aspectos relacionados con el género de las víctimas (Hightower, 2004, p. 61; Daichman, 2005, p. 325; Straka y Montminy, 2006, p. 263). A pesar de que el movimiento MPAM fue inicialmente llamado «movimiento de abuelas maltratadas» (granny battering en inglés), rara vez ha analizado la violencia experimentada por las mujeres mayores de manera particular. Como resultado, este campo de estudio ha pasado por alto las diferencias entre las experiencias de maltrato entre hombres y mujeres mayores, considerándolos una población homogénea y sin género. Ignorar esos aspectos reproduce estereotipos viejistas y desconocen el hecho de que el abuso que sufren las mujeres mayores en la vejez puede también ser una continuidad de la violencia de género que han experimentado a lo largo de sus vidas (Vinton, 1991, p. 6; Fisher et al., 2003, p. 1409; HelpAge International, 2017; Bows, 2019, p. 7).

\section{Limitaciones conceptuales y teóricas de ambos marcos}

Ambos marcos conceptuales (VCM y MPAM) tienen aspectos similares. Entienden «violencia» $\mathrm{y}$ «abuso» como el uso intencional de la fuerza física o el poder contra una persona «que resulta o tiene una alta probabilidad de resultar en lesiones, muerte, daño psicológico, mal desarrollo o privación» (OMS, 2002, p. 5). En ese sentido, puede tomar formas múltiples y concurrentes: «actos de abuso físico, abuso financiero, negligencia, abuso psicológico, agresión sexual y violaciones de los derechos humanos» (Hightower, 2004, p. 60). Además, 
manifestaron que la violencia podría ser perpetrada por alguien que tuviera una relación establecida con la víctima (Crockett et al., 2015, p. 293).

Sin embargo, ambos campos de estudio muestran diferencias en sus formas de estudiar y operacionalizar la violencia. El marco de VCM a menudo pasa por alto algunos tipos de abuso (negligencia y financieros) y de perpetradores (miembros de la familia) que suelen estar relacionados con la experiencia de violencia de las mujeres en la vejez. Sin embargo, los estudios de VCM suelen considerar con más frecuencia que los estudios de MPAM que la violencia es una experiencia que las mujeres viven a lo largo de su curso vital. Además, ambos marcos homogeneizan e invisibilizan el tema de la violencia contra las mujeres adultas mayores. Por lo tanto, descuidan el hecho de que es una problemática basada en el género y la edad simultáneamente, pero fallan en considerar las necesidades, características y perspectivas únicas de las mujeres mayores. La negligencia hacia las mujeres adultas mayores también se refleja en los servicios de protección para los adultos mayores y mujeres que fallan en detectar y abordar este tema (Band-Winterstein y Eisikovits, 2010, pp. 202-204; Hightower, 2010, pp. 17-18; Bows, 2019, pp. 9-12; Straka y Montminy, 2006).

En conclusión, ambos marcos muestran limitaciones para el análisis de la violencia contra las mujeres adultas mayores desde un abordaje como problemática de género y edad. Esta forma fragmentada de abordar la violencia, que se ha denominado «caos de definiciones» (Bows, 2019) o «superposición conceptual» (Crockett et al., 2015), no nos permite comprender plenamente la experiencia de la violencia de las mujeres mayores. Ambos marcos dividen la vida de las mujeres mayores en etapas separadas (niña, adolescente, mujer, mujer mayor) y en características (edad y género). Esto hace que sea imposible observar la conexión entre sus experiencias de violencia en su vida como un continuo a lo largo de la vida y la intersección de ambos factores de desventaja. Como resultado, estos marcos separados producen datos dispares que no pueden compararse y que presentan diferentes resultados (Brownell, 2019, pp. 2-3; Meyer et al., 2019). Además, la construcción de estos «silos» o «cajas» no solo impide comprender teóricamente las experiencias reales de las mujeres y su relación con la violencia durante el transcurso de su vida, sino que también entorpece el diálogo entre colegas para compartir conocimientos (Band-Winterstein y Eisikovits, 2009, p. 165; Hightower, 2010 , p. 25). Esto, como consecuencia, ha impedido el rápido desarrollo de la investigación en esta área de estudios. 


\section{Interseccionalidad}

Organizaciones internacionales ${ }^{4}$ e investigadores europeos han sugerido que la adopción del marco interseccional podría resolver los problemas conceptuales para abordar este tema. La interseccionalidad es un concepto originalmente acuñado por la académica feminista Kimberlé Crenshaw (1989) para explicar las experiencias particulares de violencia de las mujeres afrodescendientes. Destaca que, aunque el género es un sistema de desigualdad medular, las mujeres no son un grupo homogéneo: están cruzadas por diferentes jerarquías que les hacen experimentar distintos tipos de asimetrías simultáneamente.

En ese sentido, como señalaron Calasanti y King (2015), la violencia debe abordarse como un problema multidimensional en el que las desigualdades de raza, clase, género y vejez están ligadas a la vida de las mujeres adultas mayores y producen formas particulares de violencia. Sugieren que las mujeres adultas mayores no se jubilan de las desigualdades de género experimentadas anteriormente. La exclusión y la violencia pueden continuar agravándose en la vejez, ya que envejecer implica asumir una posición aún más desfavorecida en la sociedad. Asimismo, varios investigadores han sugerido que adoptar un enfoque de curso de vida en la investigación interseccional sobre esta temática permitiría pensar en la experiencia de la violencia como un continuo que busca conectar las diversas formas de violencia y abuso que experimentan las mujeres a través del tiempo, el lugar y el espacio. Además, permitiría identificar y mapear esta violencia a lo largo de la vida de la persona y comprender cómo la edad puede afectar algunas de las formas en que esta se manifiesta en diferentes momentos de la vida de manera interconectada (Band-Winterstein y Eisikovits, 2009, p. 165; Bows, 2019, p. 182; Brownell, 2019, p. 2; McGarry y Ali, 2019, p. 43; Mikulionienè y Tamutiené, 2019, p. 18).

Aún son pocos los investigadores de la violencia contra las mujeres adultas mayores que hayan incorporado los marcos conceptuales de interseccionalidad. Sin embargo, durante la última década, un nuevo conjunto de estudios ha mostrado indicios de una transformación. Los estudios cuantitativos — que siempre han dominado el campo - que desagregan los datos por edad y sexo cada vez son más comunes. Además, comienzan a aparecer estudios cualitativos y participativos para completar la brecha y explorar el tema a través de una perspectiva fenomenológica que dé voz a las percepciones y las historias de vida de las sobrevivientes desde

4 Este reclamo es presentado en el Plan Internacional de Acción en Envejecimiento de Madrid (MIPAA) y en la Declaración sobre la Prevención Global del Abuso de Ancianos de Toronto. 
la adopción de un enfoque narrativo de la violencia experimentada (Fisher et al., 2003, p. 1413; Mears, 2003; Zink et al., 2003; Band-Winterstein y Eisikovits, 2010, pp. 203-205; Hightower, 2010, pp. 21-23; Weeks et al., 2016).

En ALC, un grupo de académicos ha destacado la urgencia de realizar estudios sobre esta problemática social durante las últimas dos décadas. Así como en el debate presentado anteriormente, los estudios sobre la VCM y el MPAM en ALC han sido fuertemente criticados. La académica brasileña Alda Britto da Motta ha enfatizado que ambos campos han sido ciegos en cuanto a la edad y el género, respectivamente. Afirma, con tanta fuerza como Hightower, que las mujeres adultas mayores también existen, destacando que el hecho de no estudiar sus problemas las ha vuelto invisibles para las políticas sociales, enfocadas en la protección de los derechos de las mujeres y niñas en la región (Motta, 2010, p. 180; Batista y Motta, 2014, p. 39). Finalmente, este grupo de académicos también ha sugerido la adopción del marco de interseccionalidad y el enfoque de curso de vida para el estudio de la violencia contra las mujeres adultas mayores, específicamente para observar la conexión de las desigualdades de género y edad, pero también de la etnicidad y la clase social (Brownell, 2016, p. 3326). Si bien estos debates teóricos están comenzando a surgir en ALC, aún se sabe poco sobre el estado de desarrollo de este nuevo campo regional. Por lo tanto, el debate presentado en esta sección será utilizado en los siguientes capítulos para evaluar en qué medida el nuevo cuerpo de trabajo en los países de ALC ha logrado conectar las desigualdades de género y envejecimiento en el estudio de este tema.

\section{METODOLOGÍA}

Este estudio se basó en una revisión sistemática y crítica de literatura académica para analizar las aproximaciones metodológicas y conceptuales de la investigación acerca de la violencia con mujeres mayores producida en ALC con la finalidad de discutir en qué medida este grupo de trabajos han logrado conectar en sus análisis las desigualdades de género y envejecimiento simultáneamente. Es decir, utilicé parámetros y procedimientos claros para identificar, evaluar críticamente y sintetizar literatura relevante (Hawker et al., 2002, p. 1285).

\section{Estrategia de búsqueda}

Las bases de datos bibliográficas empleadas en la búsqueda de literatura fueron Web of Science (WoS Thomson Reuters), Delphis y Scopus 
(Elsevier) ${ }^{5}$. Los términos de búsqueda elegidos buscaron dar cuenta de los principales aspectos de la pregunta de investigación y se emplearon términos en inglés y español con el deseo de identificar textos escritos en la región.

\section{Tabla 1. Términos de búsqueda}

\begin{tabular}{|c|c|}
\hline En i & En español \\
\hline $\begin{array}{l}\text { Ag*ng OR elder* OR «old* person*» OR } \\
\text { «old* people» OR «old* adult*» OR «old } \\
\text { age” OR aged OR «older wom } \$ \text { n» OR older } \\
\text { OR «later life» OR } 60 \text { OR } 65 \text { [Abstract] }\end{array}$ & 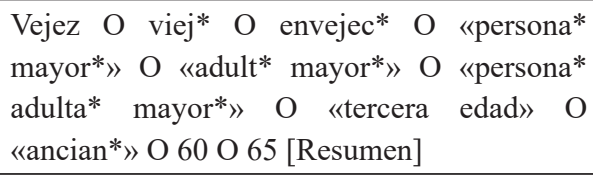 \\
\hline $\begin{array}{l}\text { Wom*n OR Gender OR «gender-based» } \\
\text { [Abstract] }\end{array}$ & Mujer* O género $\mathrm{O}$ femenin* [Resumen] \\
\hline $\begin{array}{l}\text { «Elder abuse» OR violence OR battered } \\
\text { OR «intimate partner violence» OR IPV } \\
\text { OR «violence against women» OR VAW } \\
\text { OR victim* OR perpetrator* OR «domestic } \\
\text { violence» OR «familial violence» OR «Me } \\
\text { too» [Title] }\end{array}$ & $\begin{array}{l}\text { maltrat* } \mathrm{O} \text { abus* } \mathrm{O} \text { violen* } \mathrm{O} \text { «violencia de } \\
\text { pareja» } \mathrm{O} \text { «violencia de género» } \mathrm{O} \text { «violencia } \\
\text { contra la mujer» } \mathrm{O} \text { víctima } \mathrm{O} \text { perpetrador* } \mathrm{O} \\
\text { «violencia doméstica» } \mathrm{O} \text { «violencia familiar» } \\
\mathrm{O} \text { «Ni una menos» } \mathrm{O} \text { «Me too» }[\text { Título] }\end{array}$ \\
\hline $\begin{array}{l}\text { «South America» OR «Latin America and the } \\
\text { Caribbean» OR «Latin America» OR LAC OR } \\
\text { Belize OR «Costa Rica» OR «El Salvador» } \\
\text { OR Guatemala OR Honduras OR Mexico } \\
\text { OR Nicaragua OR Panama OR Argentina OR } \\
\text { Bolivia OR Brazil OR Chile OR Colombia OR } \\
\text { Ecuador OR «French Guiana» OR Guyana OR } \\
\text { Paraguay OR Peru OR Suriname OR Uruguay } \\
\text { OR Venezuela OR Caribbean OR Cuba OR } \\
\text { «Dominican Republic» OR Haiti OR «Puerto } \\
\text { Rico» [Abstract] }\end{array}$ & $\begin{array}{l}\text { «América del Sur»O «Latino América y el } \\
\text { Caribe» O «Latino América» O ALC O Belice } \\
\text { O «Costa Rica»O «El Salvador» O Guatemala } \\
\text { O Honduras O México O Nicaragua O Panamá } \\
\text { O Argentina O Bolivia O Bra*il O Chile O } \\
\text { Colombia O Ecuador O «Guyana Francesa» } \\
\text { O Guyana O Paraguay O Perú O Suriname } \\
\text { O Uruguay O Venezuela O Caribe O Cuba O } \\
\text { «República Dominicana»O Haití O «Puerto } \\
\text { Rico» [Resumen] }\end{array}$ \\
\hline
\end{tabular}

Adicionalmente, realicé una «búsqueda creativa» (Hawker et al., 2002, p. 1287), para identificar estudios adicionales combinando las técnicas

5 Seleccioné estas bases de datos no solo por su dominio en el campo y garantía de calidad, sino también porque indexan un número considerable de revistas latinoamericanas bien establecidas Web of Science y Scopus han indexado todas las revistas latinoamericanas, incluidas en Scielo. Esta base de datos bibliográfica brasileña tiene por objeto difundir revistas iberoamericanas de alta calidad (de América Latina, España y Portugal) (Rodrigues y Abadal, 2014, p. 56). Scielo no se incluyó como base de datos separada para la búsqueda porque no permitía la ejecución de una búsqueda sistemática. 
de «bola de nieve» (Patticrew y Roberts, 2006, p. 121) —es decir, rastreando referencias citadas en artículos y libros ya identificados-, así como también búsquedas a través de Google Scholar y comunicaciones personales con investigadores y expertos en la temática.

\section{Criterios de inclusión y exclusión}

Los siguientes criterios inclusión buscaron que los estudios seleccionados: (i) hayan sido realizados en países de América Latina y el Caribe; (ii) contengan muestras que incluyan a mujeres de 60 años y más; (iii) estén relacionados con las experiencias de violencia de mujeres mayores ejercidas por cualquier tipo de perpetrador; (iv) sean empíricos basados en métodos cualitativos, cuantitativos o mixtos; excluyendo reseñas de literatura o ensayos; (v) estén publicados en revistas académicas, capítulos de libros o libros; (vi) sean de los campos disciplinarios social, sanitario, conductual o del desarrollo; (vii) estén escritos en inglés, así como en los idiomas más hablados en América Latina y el Caribe: español o portugués, y (viii) estudios publicados hasta el año 2020.

\section{CRITERIOS DE EXCLUSIÓN}

\section{Resultados y refinamiento de la búsqueda}

Después de todos estos procesos de búsqueda, identifiqué 177 artículos que fueron gestionados mediante el software «Endnote» para identificar entradas duplicadas y eliminarlas. Quedaron 159 documentos. Finalmente, evalué la calidad de los estudios mediante criterios estandarizados para decidir cuáles serían analizados. Las publicaciones seleccionadas debían: (i) presentar sus marcos conceptuales y operacionalización de violencia; (ii) hacer explícita su estrategia metodológica; (iii) describir sus técnicas y procesos de recojo de información, y (iv) desagregar sus hallazgos por sexo y grupos de edad. Luego de esta evaluación, seleccioné 72 publicaciones para ser parte del presente estudio y, para fines analíticos, han sido divididas en tres grupos dependiendo del marco teórico que adoptaron: MPAM, VCM o Interseccional.

Esta estrategia metodológica se explica en mayor detalle a continuación con el diagrama de flujo de PRISMA 2020. 


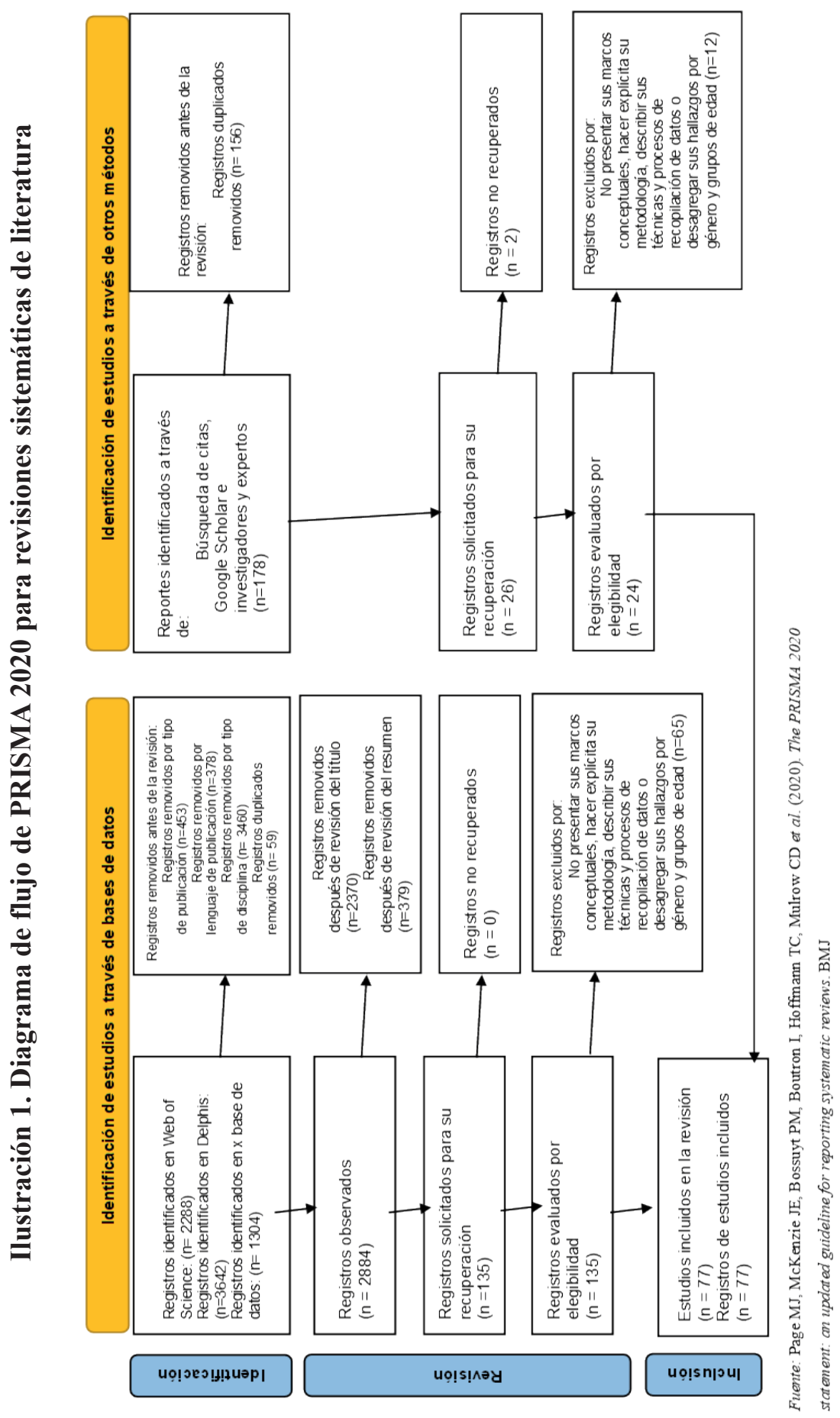




\section{RESULTADOS}

\section{Visión general del estado de desarrollo del campo}

La investigación académica sobre la violencia contra las mujeres adultas mayores en ALC aún es reciente. Sin embargo, muestra un crecimiento sostenido desde inicios de 2000 hasta la fecha, especialmente en algunos países, como Brasil y México. Como se demostrará a lo largo de este artículo, la mayoría de las publicaciones analizadas abordan la violencia contra las mujeres adultas mayores de manera superficial, ya que no están enfocadas únicamente en esta población. Estos estudios tienen como objetivo o caracterizar la MPAM o la VCM. Por tanto, las experiencias y particularidades de las mujeres adultas mayores no son su principal inquietud. Sin embargo, los estudios sobre el abuso de personas mayores que desagregan sus hallazgos por género se incrementaron. Por su parte, como evidencia la tabla 2, el campo de la VCM no parecía mostrar un interés particular en incorporar a mujeres adultas mayores de sesenta años en sus estudios, ya que, esta línea de trabajo no contiene un gran número de investigaciones que incluyan a mujeres mayores, a pesar de que los estudios de género cuentan con una larga data en la región. Finalmente, durante las décadas de 2010-2020, se observó el origen y crecimiento de un nuevo campo enfocado en las experiencias de violencia de las mujeres adultas mayores. Estos estudios adoptan un enfoque interseccional y de curso de vida.

La mayoría de las investigaciones fue desarrollada por especialistas en campos de la medicina, como salud pública, medicina humana y enfermería. No obstante, recientemente se han incrementado las investigaciones de científicos sociales de varias disciplinas, como trabajo social, psicología social y sociología, especialmente en la investigación enfocada en mujeres adultas mayores.

Por último, debido a que este el tema en cuestión es multidimensional e interdisciplinario, los 72 estudios realizados han sido publicados en 52 revistas académicas diferentes de distintos campos del conocimiento. Esta particularidad hace que su identificación como un campo consolidado sea más difícil, ya que su localización resulta más compleja. Por otro lado, fueron redactados en tres idiomas - español, inglés y portugués-, lo que podría dificultar el diálogo entre ellas. Estos estudios se muestran en las tablas 3, 4 y 5, que se presentan a continuación. 


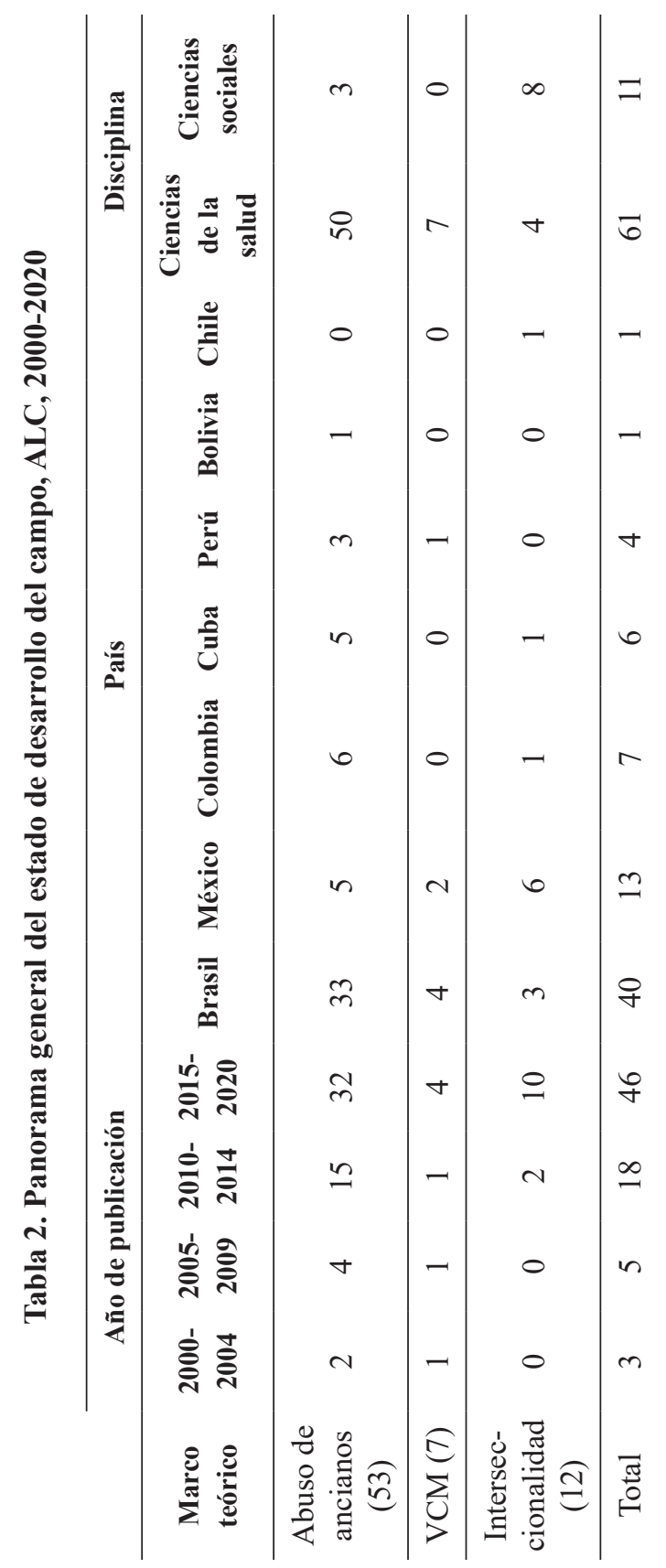


Tabla 3. Estudios realizados desde el marco teórico de MPAM

\begin{tabular}{|c|c|c|c|c|c|c|}
\hline Referencia & Objetivo & Disciplina & $\begin{array}{l}\text { Tempo- } \\
\text { ralidad }\end{array}$ & Muestra & Participantes & $\begin{array}{l}\text { Técnica de } \\
\text { recojo }\end{array}$ \\
\hline \multicolumn{7}{|c|}{ Estudios cuantitativos- probabilísticos } \\
\hline $\begin{array}{l}\text { Agudelo } \\
\text { et al. } \\
(2019)\end{array}$ & $\begin{array}{l}\text { Determinar la prevalencia del } \\
\text { maltrato a personas mayores } \\
\text { y su asociación con factores } \\
\text { sociodemográficos y familiares. }\end{array}$ & $\begin{array}{l}\text { Sistemas } \\
\text { de infor- } \\
\text { mación en } \\
\text { salud }\end{array}$ & \multirow{10}{*}{ Transversal } & 506 & $\begin{array}{l}\text { Personas } \\
\text { adultas } \\
\text { mayores } \\
\text { - ambos } \\
\text { géneros }\end{array}$ & Encuesta \\
\hline $\begin{array}{l}\text { Alencar } \\
\text { et al. } \\
(2018)\end{array}$ & $\begin{array}{l}\text { Analizar la asociación entre } \\
\text { características sociodemográ- } \\
\text { ficas, conductuales y de salud } \\
\text { y la prevalencia de la violencia } \\
\text { contra las personas mayores } \\
\text { cometida por desconocidos. }\end{array}$ & Estadística & & 11143 & & $\begin{array}{l}\text { Encuesta } \\
\text { nacional }\end{array}$ \\
\hline $\begin{array}{l}\text { Apratto } \\
(2010)\end{array}$ & $\begin{array}{l}\text { Investigar la magnitud y carac- } \\
\text { terísticas de la violencia contra } \\
\text { las personas mayores por } \\
\text { personas de confianza. }\end{array}$ & Medicina & & 343 & & \multirow{8}{*}{ Encuesta } \\
\hline $\begin{array}{l}\text { Bardales y } \\
\text { Menéndez } \\
(2015)\end{array}$ & $\begin{array}{l}\text { Explorar las características de } \\
\text { la violencia doméstica y sexual } \\
\text { que experimentan los adultos } \\
\text { mayores y su demanda de servi- } \\
\text { cios de protección. }\end{array}$ & Psicología & & 400 & & \\
\hline $\begin{array}{l}\text { Bolsoni } \\
\text { et al. } \\
(2019)\end{array}$ & $\begin{array}{l}\text { Estimar la prevalencia de la } \\
\text { violencia y su asociación con } \\
\text { las condiciones de salud de las } \\
\text { personas mayores. }\end{array}$ & $\begin{array}{l}\text { Salud } \\
\text { pública }\end{array}$ & & 1140 & & \\
\hline $\begin{array}{l}\text { Cano et al. } \\
(2015)\end{array}$ & $\begin{array}{l}\text { Determinar los factores demo- } \\
\text { gráficos, sociales y económicos } \\
\text { asociados con el abuso de } \\
\text { personas mayores. }\end{array}$ & $\begin{array}{l}\text { Salud } \\
\text { pública }\end{array}$ & & 4215 & & \\
\hline $\begin{array}{l}\text { Cardona } \\
\text { et al. } \\
(2010)\end{array}$ & $\begin{array}{l}\text { Analizar el apoyo social brin- } \\
\text { dado a los adultos mayores } \\
\text { institucionalizados. }\end{array}$ & $\begin{array}{l}\text { Salud } \\
\text { pública }\end{array}$ & & 273 & 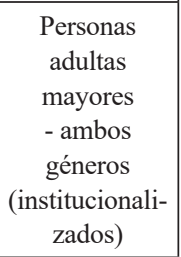 & \\
\hline $\begin{array}{l}\text { Cardoso } \\
\text { et al. } \\
(2004)\end{array}$ & $\begin{array}{l}\text { Caracterizar el maltrato en las } \\
\text { personas mayores. }\end{array}$ & Medicina & & 84 & \multirow{2}{*}{$\begin{array}{l}\text { Personas } \\
\text { adultas } \\
\text { mayores } \\
\text { - ambos } \\
\text { géneros }\end{array}$} & \\
\hline $\begin{array}{l}\text { Casanova } \\
\text { et al. } \\
(2013)\end{array}$ & $\begin{array}{l}\text { Caracterizar manifestaciones } \\
\text { de violencia doméstica hacia } \\
\text { personas mayores con diabetes. }\end{array}$ & Medicina & & 52 & & \\
\hline $\begin{array}{l}\text { Castro } \\
\text { et al. } \\
(2018)\end{array}$ & $\begin{array}{l}\text { Identificar la prevalencia de } \\
\text { agresión física y negligencia } \\
\text { y abandono y la asociación de } \\
\text { estos con variables sociodemo- } \\
\text { gráficas. }\end{array}$ & Enfermería & & 477 & $\begin{array}{l}\text { Personas } \\
\text { adultas } \\
\text { mayores } \\
\text { - ambos } \\
\text { géneros } \\
\text { (hospitali- } \\
\text { zados) }\end{array}$ & \\
\hline
\end{tabular}




\begin{tabular}{|c|c|c|c|c|c|c|}
\hline Referencia & Objetivo & Disciplina & $\begin{array}{l}\text { Tempo- } \\
\text { ralidad }\end{array}$ & Muestra & Participantes & $\begin{array}{c}\text { Técnica de } \\
\text { recojo }\end{array}$ \\
\hline $\begin{array}{l}\text { Curcio } \\
\text { et al. } \\
(2019)\end{array}$ & $\begin{array}{l}\text { Describir la presencia de } \\
\text { maltrato en personas mayores } \\
\text { y su asociación con condiciones } \\
\text { sociodemográficas y funcionales. }\end{array}$ & Medicina & & 23694 & $\begin{array}{l}\text { Personas } \\
\text { adultas } \\
\text { mayores- } \\
\text { ambos } \\
\text { géneros }\end{array}$ & $\begin{array}{l}\text { Encuesta } \\
\text { nacional }\end{array}$ \\
\hline $\begin{array}{l}\text { Duque } \\
\text { et al. } \\
(2012)\end{array}$ & $\begin{array}{l}\text { Determinar la prevalencia y los } \\
\text { factores asociados a la violencia } \\
\text { doméstica contra las personas } \\
\text { mayores. }\end{array}$ & $\begin{array}{l}\text { Salud } \\
\text { pública }\end{array}$ & & 274 & & \multirow{10}{*}{ Encuesta } \\
\hline $\begin{array}{l}\text { Faustino } \\
\text { et al. } \\
(2014)\end{array}$ & $\begin{array}{l}\text { Presentar el perfil sociodemo- } \\
\text { gráfico, e identificar los hechos } \\
\text { violentos de diversa índole } \\
\text { (físicos, psicológicos, sexuales, } \\
\text { abandono, negligencia, abuso } \\
\text { económico y autonegligencia) } \\
\text { en personas mayores. }\end{array}$ & Enfermería & & 131 & & \\
\hline $\begin{array}{l}\text { Gómez } \\
\text { et al. } \\
(2007)\end{array}$ & $\begin{array}{l}\text { Estimar la prevalencia del abuso } \\
\text { familiar de ancianos y determinar } \\
\text { los factores de riesgo asociados. }\end{array}$ & $\begin{array}{l}\text { Salud } \\
\text { pública }\end{array}$ & & 1078 & & \\
\hline $\begin{array}{l}\text { Griñan } \\
\text { et al. } \\
(2012)\end{array}$ & $\begin{array}{l}\text { Caracterizar a las víctimas de } \\
\text { maltrato familiar en este grupo } \\
\text { poblacional. }\end{array}$ & Medicina & & 300 & & \\
\hline $\begin{array}{l}\text { Guedes } \\
\text { et al. } \\
(2015)\end{array}$ & $\begin{array}{l}\text { Determinar si el género, las } \\
\text { condiciones socioeconómicas } \\
\text { o las relaciones sociales se } \\
\text { vinculan con experiencias } \\
\text { recientes de violencia domés- } \\
\text { tica. }\end{array}$ & Medicina & & 800 & & \\
\hline $\begin{array}{l}\text { Guedes } \\
\text { et al. } \\
(2015)\end{array}$ & $\begin{array}{l}\text { Describir la prevalencia de } \\
\text { la violencia doméstica en los } \\
\text { adultos mayores y explorar si } \\
\text { las diferencias por género se } \\
\text { deben a diferencias de por vida } \\
\text { en el estatus social y financiero. }\end{array}$ & $\begin{array}{l}\text { Salud } \\
\text { pública }\end{array}$ & & 802 & & \\
\hline $\begin{array}{l}\text { Júnior } \\
\text { (2010) }\end{array}$ & $\begin{array}{l}\text { Investigar la magnitud y carac- } \\
\text { terísticas de la violencia contra } \\
\text { las personas mayores por } \\
\text { personas de confianza. }\end{array}$ & Medicina & & 343 & & \\
\hline $\begin{array}{l}\text { Maia et al. } \\
\text { (2019) }\end{array}$ & $\begin{array}{l}\text { Analizar la ocurrencia de } \\
\text { violencia en el adulto mayor y } \\
\text { sus factores asociados. }\end{array}$ & Medicina & & 178 & & \\
\hline $\begin{array}{l}\text { Mancinas } \\
\text { y Ribeiro } \\
(2010)\end{array}$ & $\begin{array}{l}\text { Identificar los factores de riesgo } \\
\text { asociados con el maltrato de } \\
\text { personas mayores en el contexto } \\
\text { familiar. }\end{array}$ & $\begin{array}{c}\text { Trabajo } \\
\text { social }\end{array}$ & & 952 & & \\
\hline $\begin{array}{l}\text { Carmona } \\
\text { et al. } \\
(2018)\end{array}$ & $\begin{array}{l}\text { Determinar el grado de abuso de } \\
\text { personas mayores y vulnerables } \\
\text { en las familias y comunidades. }\end{array}$ & Enfermería & & 210 & & \\
\hline
\end{tabular}




\begin{tabular}{|c|c|c|c|c|c|c|}
\hline Referencia & Objetivo & Disciplina & $\begin{array}{l}\text { Tempo- } \\
\text { ralidad }\end{array}$ & Muestra & Participantes & $\begin{array}{l}\text { Técnica de } \\
\text { recojo }\end{array}$ \\
\hline \begin{tabular}{|l} 
Moraes \\
et al. \\
$(2008)$
\end{tabular} & $\begin{array}{l}\text { Estudiar la prevalencia de la } \\
\text { violencia física doméstica } \\
\text { contra las personas mayores. }\end{array}$ & Medicina & & 332 & & \multirow{4}{*}{ Encuesta } \\
\hline $\begin{array}{l}\text { Paiva y } \\
\text { Tavares } \\
(2015)\end{array}$ & $\begin{array}{l}\text { Verificar la prevalencia } \\
\text { y factores asociados con la } \\
\text { violencia física y psicológica } \\
\text { contra las personas mayores y } \\
\text { rastrear los indicadores socio- } \\
\text { demográficos y clínicos de esta } \\
\text { población. }\end{array}$ & Medicina & & 729 & & \\
\hline $\begin{array}{l}\text { Ruelas } \\
\text { et al. } \\
(2016)\end{array}$ & $\begin{array}{l}\text { Identificar la prevalencia y } \\
\text { los factores asociados con la } \\
\text { violencia y el abuso de los } \\
\text { adultos mayores. }\end{array}$ & $\begin{array}{l}\text { Salud } \\
\text { pública }\end{array}$ & & 8894 & & \\
\hline $\begin{array}{l}\text { Silva et al. } \\
\text { (2015) }\end{array}$ & $\begin{array}{l}\text { Identificar la prevalencia, tipos } \\
\text { de violencia intrafamiliar, } \\
\text { su asociación con variables } \\
\text { sociodemográficas y síntomas } \\
\text { depresivos en adultos mayores. }\end{array}$ & Enfermería & & 369 & & \\
\hline \multicolumn{7}{|c|}{ Estudios cuantitativos no probabilísticos (encuestas) } \\
\hline $\begin{array}{l}\text { Lino et al. } \\
(2019)\end{array}$ & $\begin{array}{l}\text { Identificar la prevalencia y los } \\
\text { factores asociados a la violencia } \\
\text { familiar perpetrada por los } \\
\text { cuidadores contra las personas } \\
\text { mayores dependientes. }\end{array}$ & Medicina & \multirow{5}{*}{ Transversal } & 135 & $\begin{array}{l}\text { Personas } \\
\text { adultas } \\
\text { mayores- } \\
\text { ambos } \\
\text { géneros (con } \\
\text { discapacidad) }\end{array}$ & \multirow{5}{*}{ Encuesta } \\
\hline $\begin{array}{l}\text { Enamorado } \\
\text { Pina et al. } \\
(2013)\end{array}$ & $\begin{array}{l}\text { Caracterizar a los adultos } \\
\text { mayores que sufrieron abuso } \\
\text { doméstico. }\end{array}$ & Medicina & & 75 & \multirow{3}{*}{$\begin{array}{l}\text { Personas } \\
\text { adultas } \\
\text { mayores- } \\
\text { ambos } \\
\text { géneros }\end{array}$} & \\
\hline $\begin{array}{l}\text { Ribot et al. } \\
(2015)\end{array}$ & $\begin{array}{l}\text { Caracterizar los casos de } \\
\text { maltrato a personas mayores } \\
\text { determinando las principales } \\
\text { manifestaciones de maltrato y } \\
\text { comportamiento de búsqueda } \\
\text { de ayuda. }\end{array}$ & Medicina & & 197 & & \\
\hline $\begin{array}{l}\text { Ruelas y } \\
\text { Salgado } \\
(2009)\end{array}$ & $\begin{array}{l}\text { Identificar algunos factores } \\
\text { sociodemográficos, en salud } \\
\text { física y mental, apoyo social y } \\
\text { utilización de los servicios de } \\
\text { salud, asociados con la autode- } \\
\text { claración de abuso. }\end{array}$ & $\begin{array}{l}\text { Salud } \\
\text { pública }\end{array}$ & & 799 & & \\
\hline $\begin{array}{l}\text { Vilar y } \\
\text { Gaitan } \\
(2018)\end{array}$ & $\begin{array}{l}\text { Identificar diferentes formas } \\
\text { de medir la gravedad y } \\
\text { la frecuencia del abuso de } \\
\text { personas mayores y evaluar si } \\
\text { las diferentes experiencias de } \\
\text { gravedad y frecuencia sugieren } \\
\text { relaciones sindémicas. }\end{array}$ & Psiquiatría & & 534 & $\begin{array}{l}\text { Solo adultas } \\
\text { mujeres }\end{array}$ & \\
\hline
\end{tabular}


Tabla 4. Estudios realizados desde el marco teórico de VCM

\begin{tabular}{|c|c|c|c|c|c|c|}
\hline Referencia & Objetivos & Disciplina & $\begin{array}{c}\text { Tempora- } \\
\text { lidad }\end{array}$ & Muestra & $\begin{array}{c}\text { Partici- } \\
\text { pantes }\end{array}$ & $\begin{array}{c}\text { Técnica } \\
\text { de recojo }\end{array}$ \\
\hline \multicolumn{7}{|c|}{ Estudios cuantitativos no probabilísticos (encuesta) } \\
\hline $\begin{array}{l}\text { Díaz et al. } \\
\text { (2002) }\end{array}$ & $\begin{array}{l}\text { Investigar la incidencia } \\
\text { y los antecedentes } \\
\text { de maltrato entre las } \\
\text { mujeres y buscar posi- } \\
\text { bles factores de riesgo } \\
\text { y asociaciones con los } \\
\text { síntomas de presenta- } \\
\text { ción. }\end{array}$ & Medicina & Transversal & 1780 & $\begin{array}{l}\text { Mujeres - } \\
\text { incluye } \\
\text { mujeres } \\
\text { mayores }\end{array}$ & Encuesta \\
\hline \multicolumn{7}{|c|}{ Estudios cuantitativos no probabilísticos (registros administrativos) } \\
\hline $\begin{array}{l}\text { Andrade } \text { et al. } \\
\text { (2016) }\end{array}$ & $\begin{array}{l}\text { Caracterizar a las } \\
\text { víctimas y casos de } \\
\text { maltrato, calcular } \\
\text { morbilidad y morta- } \\
\text { lidad. }\end{array}$ & Medicina & \multirow{5}{*}{ Transversal } & 19213 & \multirow{5}{*}{$\begin{array}{l}\text { Mujeres - } \\
\text { incluye } \\
\text { mujeres } \\
\text { mayores }\end{array}$} & $\begin{array}{c}\text { Data } \\
\text { secundaria- } \\
\text { Registros } \\
\text { de salud }\end{array}$ \\
\hline $\begin{array}{l}\text { Bernardino } \\
\text { et al. (2016) }\end{array}$ & $\begin{array}{l}\text { Describir el perfil de } \\
\text { la violencia contra la } \\
\text { mujer en las distintas } \\
\text { etapas de la vida, según } \\
\text { las características socio- } \\
\text { demográficas de las } \\
\text { víctimas y agresores. }\end{array}$ & $\begin{array}{l}\text { Salud } \\
\text { pública }\end{array}$ & & 1388 & & \multirow{2}{*}{$\begin{array}{c}\text { Data } \\
\text { secundaria- } \\
\text { Registros } \\
\text { policiales }\end{array}$} \\
\hline $\begin{array}{l}\text { Curro et al. } \\
\text { (2017) }\end{array}$ & $\begin{array}{l}\text { Determinar la inci- } \\
\text { dencia de violencia } \\
\text { extrema contra la mujer } \\
\text { y feminicidio; y algunos } \\
\text { factores de riesgo. }\end{array}$ & $\begin{array}{l}\text { Salud } \\
\text { pública }\end{array}$ & & 1111 & & \\
\hline $\begin{array}{l}\text { Garcia et al. } \\
\text { (2016) }\end{array}$ & $\begin{array}{l}\text { Identificar factores } \\
\text { asociados a la atención } \\
\text { de la violencia domés- } \\
\text { tica y familiar entre las } \\
\text { víctimas. }\end{array}$ & Medicina & & 623 & & $\begin{array}{c}\text { Data } \\
\text { secundaria- } \\
\text { Registros } \\
\text { de salud }\end{array}$ \\
\hline $\begin{array}{l}\text { Leite } \text { et al. } \\
\text { (2014) }\end{array}$ & $\begin{array}{l}\text { Caracterizar a las } \\
\text { víctimas y casos de } \\
\text { abuso. }\end{array}$ & Medicina & & 7487 & & $\begin{array}{c}\text { Data } \\
\text { secundaria- } \\
\text { Registros } \\
\text { policiales }\end{array}$ \\
\hline \multicolumn{7}{|c|}{ Estudios cualitativos } \\
\hline $\begin{array}{l}\text { Agoff, C. et al. } \\
(2006)\end{array}$ & $\begin{array}{l}\text { Identificar factores } \\
\text { personales, culturales } \\
\text { e institucionales que } \\
\text { dificultan la solución de } \\
\text { la violencia doméstica. }\end{array}$ & $\begin{array}{l}\text { Salud } \\
\text { pública }\end{array}$ & Transversal & 26 & $\begin{array}{l}\text { Mujeres - } \\
\text { incluye } \\
\text { mujeres } \\
\text { mayores }\end{array}$ & Entrevistas \\
\hline
\end{tabular}


Tabla 5. Estudios interseccionales

\begin{tabular}{|c|c|c|c|c|c|c|}
\hline Referencia & Objetivos & Disciplina & $\begin{array}{c}\text { Tempora- } \\
\text { lidad }\end{array}$ & Muestra & $\begin{array}{l}\text { Partici- } \\
\text { pantes }\end{array}$ & \begin{tabular}{|c|} 
Técnica de \\
recojo
\end{tabular} \\
\hline \multicolumn{7}{|c|}{ Estudios cuantitativos probabilísticos (encuesta nacional) } \\
\hline $\begin{array}{l}\text { Frías S. } \\
\text { (2016) }\end{array}$ & $\begin{array}{l}\text { Describir las experiencias de } \\
\text { violencia y polivictimización } \\
\text { de mujeres mayores en México } \\
\text { y sus factores asociados. }\end{array}$ & $\begin{array}{l}\text { Ciencias } \\
\text { sociales }\end{array}$ & & 20940 & & \\
\hline $\begin{array}{l}\text { Rodríguez } \\
\text { y Esquivel } \\
(2020)\end{array}$ & $\begin{array}{l}\text { Examinar la prevalencia y } \\
\text { los factores asociados con la } \\
\text { victimización por violencia de } \\
\text { pareja íntima (VPI) emocional, } \\
\text { física y sexual entre una muestra } \\
\text { representativa de mujeres mexi- } \\
\text { canas mayores, utilizando un } \\
\text { enfoque ecológico. }\end{array}$ & $\begin{array}{l}\text { Ciencias } \\
\text { sociales }\end{array}$ & $\begin{array}{l}\text { Trans- } \\
\text { versal }\end{array}$ & 7410 & $\begin{array}{l}\text { Mujeres } \\
\text { mayores }\end{array}$ & $\begin{array}{c}\text { Data } \\
\text { secundaria }\end{array}$ \\
\hline \multicolumn{7}{|c|}{ Estudios cuantitativos no probabilísticos (encuesta) } \\
\hline $\begin{array}{l}\text { Vilar et al. } \\
\text { (2017) }\end{array}$ & $\begin{array}{l}\text { Evaluar la asociación entre } \\
\text { depresión y maltrato a personas } \\
\text { mayores, y el efecto mediador } \\
\text { del apoyo social entre mujeres } \\
\text { mayores en la Ciudad de } \\
\text { México. }\end{array}$ & $\begin{array}{l}\text { Salud } \\
\text { pública }\end{array}$ & $\begin{array}{l}\text { Trans- } \\
\text { versal }\end{array}$ & 526 & $\begin{array}{l}\text { Mujeres } \\
\text { mayores }\end{array}$ & Encuesta \\
\hline \multicolumn{7}{|c|}{ Estudios cualitativos } \\
\hline $\begin{array}{l}\text { Bruno y } \\
\text { Castro } \\
(2019)\end{array}$ & $\begin{array}{l}\text { Contribuir con evidencia } \\
\text { empírica, desde una discusión } \\
\text { teórica, sobre la relación entre } \\
\text { vejez, violencia, redes de apoyo, } \\
\text { desde la perspectiva del cons- } \\
\text { truccionismo social. }\end{array}$ & $\begin{array}{l}\text { Ciencias } \\
\text { sociales }\end{array}$ & $\begin{array}{l}\text { Trans- } \\
\text { versal }\end{array}$ & 1 & $\begin{array}{l}\text { Mujeres } \\
\text { mayores }\end{array}$ & $\begin{array}{l}\text { Historia } \\
\text { de vida }\end{array}$ \\
\hline $\begin{array}{l}\text { Fernandes } \\
\text { y Garcia } \\
(2010)\end{array}$ & $\begin{array}{l}\text { Analizar la percepción y expe- } \\
\text { riencia de las mujeres mayores } \\
\text { sobre su cuerpo, considerando } \\
\text { la perspectiva de género. }\end{array}$ & $\begin{array}{l}\text { Ciencias } \\
\text { sociales }\end{array}$ & & 18 & & $\begin{array}{l}\text { Entrevistas } \\
\mathrm{y} \\
\text { workshops }\end{array}$ \\
\hline $\begin{array}{l}\text { Hirt et al. } \\
\text { (2018) }\end{array}$ & $\begin{array}{l}\text { Comprender las representa- } \\
\text { ciones sociales de la violencia } \\
\text { contra la mujer rural, desde } \\
\text { la perspectiva de las personas } \\
\text { mayores, considerando cómo la } \\
\text { generación y el género influyen } \\
\text { en esta agravación. }\end{array}$ & Enfermería & & $\begin{array}{l}12 \text { rural } \\
\text { women }\end{array}$ & & $\begin{array}{c}\text { Entrevistas } \\
\mathrm{y} \\
\text { workshops }\end{array}$ \\
\hline
\end{tabular}




\begin{tabular}{|c|c|c|c|c|c|c|}
\hline Referencia & Objetivos & Disciplina & $\begin{array}{c}\text { Tempora- } \\
\text { lidad }\end{array}$ & Muestra & $\begin{array}{c}\text { Partici- } \\
\text { pantes }\end{array}$ & \begin{tabular}{|c|} 
Técnica de \\
recojo
\end{tabular} \\
\hline $\begin{array}{l}\text { Jiménez } \\
\text { (2012) }\end{array}$ & $\begin{array}{l}\text { Analizar las percepciones sobre } \\
\text { la violencia de las abuelas } \\
\text { abusadas por sus nietos y } \\
\text { describir las formas que la } \\
\text { violencia impacta en su vida } \\
\text { diaria. }\end{array}$ & $\begin{array}{c}\text { Trabajo } \\
\text { social }\end{array}$ & & 12 & & Entrevistas \\
\hline $\begin{array}{l}\text { Cartagena } \\
\text { y Curcio } \\
(2019)\end{array}$ & $\begin{array}{l}\text { Analizar las experiencias de } \\
\text { abuso de personas mayores a } \\
\text { través de una lente de género. }\end{array}$ & $\begin{array}{l}\text { Ciencias } \\
\text { sociales }\end{array}$ & & 8 & $\begin{array}{l}\text { Personas } \\
\text { mayores- } \\
\text { ambos } \\
\text { géneros }\end{array}$ & \multirow[b]{2}{*}{ Entrevistas } \\
\hline $\begin{array}{l}\text { López y } \\
\text { Carrasco } \\
(2013)\end{array}$ & $\begin{array}{l}\text { Identificar al abusador, el tipo } \\
\text { de violencia, el contexto social } \\
\text { del abuso y las formas en que } \\
\text { las mujeres mayores responden } \\
\text { al abuso. }\end{array}$ & $\begin{array}{l}\text { Ciencias } \\
\text { sociales }\end{array}$ & & 131 & $\begin{array}{l}\text { Mujeres } \\
\text { mayores }\end{array}$ & \\
\hline $\begin{array}{l}\text { Orbea } \\
(2017)\end{array}$ & $\begin{array}{l}\text { Analizar cómo se presenta } \\
\text { el problema de la violencia } \\
\text { de pareja íntima en un grupo } \\
\text { poblacional de mujeres mayores } \\
\text { de sesenta años (factores de } \\
\text { riesgo y características de las } \\
\text { víctimas). }\end{array}$ & $\begin{array}{c}\text { Ciencias } \\
\text { sociales }\end{array}$ & & 21 & & $\begin{array}{c}\text { Encuestas } \\
\mathrm{y} \\
\text { entrevistas }\end{array}$ \\
\hline $\begin{array}{l}\text { Sepúlveda } \\
\text { (2016) }\end{array}$ & $\begin{array}{l}\text { Analizar las creencias y tradi- } \\
\text { ciones religiosas de las mujeres } \\
\text { mayores que sufren abuso y su } \\
\text { relación con las barreras en la } \\
\text { búsqueda de ayuda. }\end{array}$ & $\begin{array}{l}\text { Ciencias } \\
\text { sociales }\end{array}$ & & 21 & & \multirow[t]{2}{*}{ Entrevistas } \\
\hline $\begin{array}{l}\text { Souto et al. } \\
\text { (2015) }\end{array}$ & $\begin{array}{l}\text { Comprender la experiencia de la } \\
\text { violencia doméstica psicológica } \\
\text { de las mujeres adultas mayores. }\end{array}$ & Enfermería & & 11 & & \\
\hline
\end{tabular}

\section{Marcos conceptuales}

Los resultados de la presente investigación muestran que el desarrollo del campo de estudio de la violencia contra las mujeres adultas mayores en ALC se ha dominado por los marcos conceptuales de MPAM y VCM. Estas investigaciones se llevaron a cabo desde una perspectiva médica o epidemiológica y estuvo enfocada en poblaciones más amplias, como todos los adultos mayores o todas las mujeres adultas en general.

Ambos campos de investigación manifestaron una incapacidad de estudiar el tema simultáneamente como un problema basado tanto en el género como en la edad. En lugar de ello, el análisis de uno de estos sistemas de desigualdad 
(sexismo o viejismo) fue priorizado, considerando al otro como uno más de los otros numerosos factores de riesgo de sufrir violencia, como la etnicidad, el nivel educativo, la situación económica o el estado de salud de la víctima. Se debe resaltar que, paradójicamente, los estudios sobre el MPAM en ALC han mostrado más interés que los estudios de VCM por explicar las causas de la violencia que las mujeres adultas mayores experimentan.

Empleando el enfoque de maltrato y abuso a las personas mayores (Naciones Unidas, 2013), los investigadores en MPAM interpretaron que las mujeres adultas mayores tenían un mayor riesgo de sufrir violencia y abuso que los hombres mayores debido a que ellas viven más y presentan mayores problemas de salud en la vejez. Ellos argumentaron que el hecho de vivir más tiempo hacía que las mujeres necesitaran de mayores cuidados, lo que representaba una carga para sus hijas e hijos adultos. En menor medida, este grupo de estudios explicaba este problema como resultado de las desigualdades de género presentes en la sociedad y de las dinámicas familiares. Sin embargo, ninguna de estas explicaciones se basa en evidencia producida por sus estudios, debido a que no presentaron información acerca de sus estados de salud o exploraron el impacto de la desigualdad de género en sus muestras. Es interesante que los estudios de VCM casi no hayan presentado ninguna explicación acerca de la violencia contra las mujeres adultas mayores usando un enfoque de género. En cambio, algunos de los estudios de VCM atribuyeron esta problemática a la sobrecarga generada por el cuidado y utilizaron el marco conceptual de MPAM.

Desde inicios de la década pasada, un nuevo grupo de estudios enfocado en mujeres adultas mayores ha emergido en algunos países de ALC — Brasil, México, Colombia, Cuba y Chile-, así como pasó en el hemisferio norte. Sin embargo, se ha prestado muy poca atención a este tema en la mayoría de países de la región. Las más recientes investigaciones que abordan el tema a través del marco interseccional y que han sido realizadas en su mayoría por científicos sociales exploran este tema a través de un lente fenomenológico y de curso de vida. Este grupo buscó identificar y mapear las experiencias de violencia de las mujeres adultas mayores durante el transcurso de su vida y comprender cómo es que el envejecimiento afecta las formas en las que la violencia y el abuso se manifiestan en la vejez.

Mi investigación reveló que este grupo de investigaciones «interseccionales» introdujo el concepto de «polivictimización» para abordar esta problemática como un continuo acumulativo que trata de conectar las diversas formas de violencia y abuso que las mujeres experimentan a través del tiempo, el lugar y es espacio, 
perpetradas por un amplio rango de actores. Adicionalmente, el uso del marco interseccional permitió a este grupo considerar los orígenes macrosociales de la VCM y no basarse únicamente en causas biológicas o individuales (estado de salud de la víctima) que buscan culpar a la víctima, tal como se le criticó al marco conceptual de MPAM (Brandl, 2006, p. 40). Por ejemplo, este grupo de estudios exploró los sistemas de creencias con las que las mujeres adultas mayores estaban socializadas para identificar que ellas justificaban las desigualdades y violencia de género que sufrían. Estos estudios describen cómo estas ideas dieron forma a sus identidades como mujeres e interactuaron con sus dinámicas familiares y maritales. A su vez, estas creencias y dinámicas presentan dificultades para denunciar la violencia o dejar a una pareja abusiva.

Estos distintos grupos de estudios - que toman marcos teóricos de VCM, MPAM e interseccional — también presentaron diferentes intereses de investigación y formas de operacionalizar sus variables de estudio. Los estudios de MPAM y VCM buscan revelar la prevalencia de la violencia, describir el tipo de abuso sufrido, identificar factores de riesgo, describir las características de las víctimas y perpetradores y detectar dificultades en la búsqueda de ayuda. Sin embargo, los estudios de MPAM han incluido una gama más amplia de perpetradores y de tipos de violencia. Por el contrario, los estudios de VCM suelen enfocarse en la violencia perpetrada por parejas en el ámbito doméstico. Estos no suelen considerar ciertos tipos de violencia - financiera y de negligencia-, con lo que pasan por alto aspectos críticos de la experiencia de abuso de mujeres en su vejez, como fue puntualizado anteriormente por las Naciones Unidas (2013).

En comparación, los estudios de interseccionalidad añaden un nuevo espectro de temas que no habían sido explorados previamente en la región. Ellos abordan la interconexión y la concurrencia de los diversos tipos de violencia durante el transcurso de la vida. Además, examinan los sistemas de creencias de las mujeres adultas mayores, exploran sus percepciones y experiencias de violencia, identifican el impacto del abuso en su estado de salud y sus cuerpos y describen cómo las víctimas crean y hacen uso de redes de soporte comunitario y familiar para enfrentar y sobrevivir a la violencia. Sin embargo, tal como criticó la académica mexicana Frías (2016, p. 368), este grupo de investigadores estuvo centrado principalmente en la violencia perpetrada por las parejas y aún existe mucha incertidumbre concerniente al abuso proveniente de otros miembros de la familia y no familiares, como los hijos adultos o cuidadores formales. 


\section{Aproximaciones metodológicas}

En cuanto a sus aproximaciones metodológicas, la mayoría de estudios sobre MPAM y VCM emplean diseños de investigación cuantitativa. En comparación, la mayoría de los estudios interseccionales enfocados en mujeres adultas mayores son cualitativos. Las ventajas y desventajas del uso de estas metodologías para el estudio de la violencia contra las mujeres adultas mayores serán discutidas a continuación.

Una de las debilidades de los estudios cuantitativos analizados es que la mayoría no brinda datos representativos sobre la magnitud y características de la violencia contra mujeres adultas mayores en ALC. La mayor parte de estos estudios eran no probabilísticos y se basaron en muestras de pequeña escala o en registros administrativos de servicios de protección social. Por lo tanto, la información obtenida por este tipo de estudios no representa el universo de mujeres adultas mayores que sufren violencia, sino una pequeña fracción de las víctimas que logró denunciar estos casos. A pesar de que hay un grupo reducido de investigaciones cuantitativas que aplica estrategias de muestreo probabilístico, la mayoría solamente tienen representatividad local (distrito o comunidad), debido a que realizaron encuestas de pequeña escala.

Algunos de los estudios de MPAM cuantitativos tuvieron representatividad nacional, como los conducidos por Alencar y Moraes (2018) y Curcio et al. (2019), los cuales buscaron analizar la asociación entre las características sociodemográficas, conductuales y sanitarias y la prevalencia del MPAM comparando los resultados por sexo utilizando los datos de la Encuesta Nacional de Salud 2013 en Brasil y la Encuesta en Salud, Bienestar y Envejecimientos en Colombia (SABE) respectivamente. Sin embargo, la mayoría de estudios tiene representatividad regional o local (e.g. Agudelo et al., sin fecha; Faustino et al., 2014; Bolsoni et al., 2019). La otra mitad de estos estudios utilizó un muestreo no probabilístico. Algunos de ellos aplicaron encuestas a pequeña escala a un reducido grupo de sujetos según su disponibilidad en espacios médicos, como los estudios descriptivos de Enamorado Pina et al. (2013) o Ribot et al. (2015), que tuvieron como objetivo identificar el tipo de maltrato sufrido, las características de las víctimas y la búsqueda de ayuda. Ambos estudios se realizaron en facultades de Medicina en Cuba y se aplicaron encuestas a 75 y 197 pacientes adultos mayores que sufrieron abuso, respectivamente. Sin embargo, la mayoría de estos estudios se basaron en denuncias de violencia o registros administrativos proporcionados por servicios de protección, como la Policía, los centros de emergencia o los servicios 
sociales y de salud (Andrade et al., 2016; Bernardino et al., 2016; Curro et al., 2017; Garcia et al., 2016; Leite et al., 2014). Solo uno de estos estudios aplicó una encuesta a un pequeño grupo de participantes reclutados en hospitales y clínicas (Díaz et al., 2002).

Por lo tanto, hay una brecha de conocimiento que debe ser resuelta mediante la realización de estudios cuantitativos representativos de la población nacional en el futuro. Lamentablemente, la mayoría de países en la región presenta limitaciones al respecto, puesto que hay una falta de este tipo de encuestas sobre MPAM y de encuestas sobre VCM que incluyan a mujeres adultas mayores. Por ello, la contribución de estos estudios cuantitativos está limitada a proporcionar pistas acerca de la existencia y las características de este problema.

Otra limitación de este grupo de estudios fue su enfoque asincrónico de la violencia. En estos no se permite observar las conexiones entre las experiencias de abuso a lo largo de la vida. Este es un problema común en ALC pues la investigación longitudinal es escasa debido a su costo comparado con los estudios transversales. Sin embargo, los «estudios interseccionales» cuantitativos muestran una novedosa y creativa forma de manejar este problema. A pesar de no ser longitudinal, la investigación de Frías (2016, p. 346) usa el concepto de polivictimización para examinar las experiencias de violencia sufridas por las participantes desde que eran niñas hasta el presente y no solo desde que cumplieron sesenta años, como se había visto en los estudios de MPAM. Para examinar la presencia de violencia pasada o actual, emplearon indicadores como «presencia de abuso infantil»o «violencia a lo largo de la vida». Esto les permite verificar su co-ocurrencia con experiencias de violencia presentes. Estos tipos de ejemplos demuestran que la posibilidad de tomar una perspectiva de curso de vida en el estudio de violencia contra las mujeres adultas mayores es viable.

Estudios interseccionales cualitativos mostraron una cantidad significativa de ventajas para el estudio de la violencia contra mujeres adultas mayores en ALC. La combinación de diversas técnicas de investigación, como entrevistas a profundidad (Bruno and Castro, 2019; Jiménez, 2012; Cartagena and Curcio, 2019; López and Carrasco, 2013; Orbea, 2017; Sepúlveda, 2016; Souto et al., 2015) o metodologías prospectivas y participativas (Fernandes and Garcia, 2010; Hirt et al., 2018), permiten la incorporación de las voces de las víctimas. Tal como Frías (2016) ha señalado, el uso de las entrevistas narrativas permitió explorar las continuidades y transformaciones de la violencia desde un enfoque de curso de vida. Sin embargo, la limitación principal de este método es que resulta imposible generalizar los resultados para todos los países de la región, pues este 
tipo de métodos tienen como objetivo la profundización. Por ello, es necesario desarrollar más estudios cualitativos que exploren las diferentes experiencias y percepciones de la violencia de las mujeres adultas mayores que viven en diferentes contextos culturales de la región.

Esta revisión crítica de la literatura ha demostrado que la mayoría de las investigaciones sobre este tema en ALC se han basado en muestras de mujeres adultas mayores autovalentes y no han tomado en cuenta a quienes son dependientes y se encuentran institucionalizadas. Solo dos estudios cuantitativos sobre el MPAM se centraron en la población que vivía en residencias a largo plazo y hospitales en Brasil (Cardona et al., 2010) y Colombia (Castro et al., 2018). Por lo tanto, es necesario y urgente utilizar el marco interseccional para estudiar otros grupos de mujeres adultas mayores que viven distintas formas de desigualdad en ALC. Por ejemplo, como propuso Brownell (2016), es necesario estudiar las experiencias de violencia de las mujeres adultas mayores con problemas de salud física o mental, así como la situación de aquellas que viven en pobreza, en zonas rurales o de distintas identidades étnicas.

\section{Hallazgos principales}

En cuanto a los principales hallazgos de estas investigaciones, los estudios sobre MPAM y VCM solo proporcionaron información limitada y superficial con relación a las mujeres adultas mayores. Las investigaciones de MPAM compararon por género el número de casos reportados en los servicios de protección y generalmente concluyeron que las mujeres adultas mayores representaban la mayoría de denuncias. Estas sumaban el 50\% a 60\% de las víctimas de abuso reportadas en Brasil y Perú (Abath et al., 2010; Martina et al., 2010; Mascarenhas et al., 2012; Garbin et al., 2016; Avanci et al., 2017; Alarcon et al., 2019; Meirelles et al., 2019). De manera similar, los estudios de VCM simplemente compararon denuncias de violencia de género por grupos de edad. Así, concluyeron que los casos de abuso contra mujeres adultas mayores representaban una pequeña fracción (de 4\% a 6\%) del total, comparadas con las mujeres en edad reproductiva.

Estos hallazgos deben tratarse con precaución, ya que no proporcionan evidencia suficiente para concluir que los hombres mayores no sufren tanta violencia como las mujeres adultas mayores, o que las víctimas de violencia doméstica son en su mayoría mujeres jóvenes. Como han subrayado anteriormente varios expertos, este tipo de resultados podría reforzar el mito común de que las víctimas de la VCM son madres jóvenes que viven con parejas abusivas y que 
la vejez es un «factor de protección» contra el abuso. No obstante, solo puede concluirse que hay ciertos grupos de la población que usan más los servicios de protección que otros. Como algunos estudios han advertido, los hombres podrían estar menos acostumbrados a denunciar la violencia que sufren, o las mujeres adultas mayores podrían experimentar más dificultades para identificar y reportar sus casos de abuso que sus contrapartes más jóvenes.

Estas hipótesis deben ser investigadas a profundidad, especialmente desde que algunos investigadores han obtenido resultados contradictorios que sugieren que, aunque las mujeres adultas mayores sufren más violencia doméstica que los hombres, los hombres mayores podrían correr más riesgo de sufrir abusos físicos perpetrados por desconocidos en espacios públicos (Abath et al., 2010, p. 1802; Sousa et al., 2016, p. 58; Bardales and Menéndez, 2015; Bolsoni et al., 2019, Castro et al., 2018; Faustino et al., 2014; Guedes et al., 2015; Ruelas et al., 2016; Gaioli and Rodrigues, 2008).

Por lo tanto, como señalé previamente, existe una necesidad urgente por desarrollar estudios cuantitativos que empleen muestras representativas para contar con información fiable en la prevalencia y magnitud de la violencia contra mujeres y hombres mayores en países de ALC. Por el contrario, sugiero limitar las investigaciones que analizan registros administrativos solo para examinar la atención prestada por los servicios de protección y las barreras para denunciar la violencia.

Además, algunos de estos estudios indicaron que las mujeres adultas mayores no experimentan la violencia como un incidente aislado. En su lugar, ellas han sufrido abusos durante varios años o décadas. Por ejemplo, un estudio no probabilístico en el Perú mostró que el $71 \%$ de mujeres que fueron víctimas de abuso de ancianos había sufrido violencia por un periodo de entre uno y diez años, 12\% por once a veinte años y un 17\% por más de veintiún años (Martina et al., 2010). De forma similar, un estudio en Brasil basado en una encuesta de pequeña escala mostró que el $62 \%$ de las mujeres adultas mayores víctimas habían sido víctimas de violencia durante el curso de su vida (Mascarenhas et al., 2012). Esos aspectos se describen con más detalle en estudios cuantitativos centrados en mujeres adultas mayores, como los estudios de Frías (2016) y Rodríguez y Esquivel (2020) en México, que usaron la encuesta poblacional sobre violencia contra las mujeres - ENDIREH, en el que se demostró que el hecho de haber sufrido o sido testigo de violencia familiar en otras etapas de la vida aumenta la probabilidad de experimentar violencia en la vejez. Frías mostró que esas experiencias previas duplicaban la tasa de prevalencia de la violencia durante la vejez, y Rodríguez y 
Esquivel indicaron que el $20 \%$ de las mujeres adultas mayores que presentaron violencia durante su vida también experimentaron abusos durante el último año.

Además, estudios cuantitativos han mostrado que la violencia contra mujeres adultas mayores se transforma a través del curso de vida y que generalmente esta es perpetrada por varios actores en simultáneo. Como se muestra en la tabla 6, una investigación realizada por Leite et al. (2014) en Brasil evidencia que las niñas y adolescentes que reportan violencia usualmente también habían sido abusadas tanto por sus parejas $(25,2 \%)$ como por miembros de su familia (24,9\%). Durante la adultez, los principales perpetradores de violencia son sus parejas $(50,8 \%)$ mientras que la violencia de otros familiares se reduce $(17,1 \%)$. Sin embargo, esto se transforma en la vejez, cuando la mayoría de los abusos provienen de familiares $(52,1 \%) \mathrm{y}$, en menor medida, de la pareja $(12,9 \%)$. Por otro lado, el estudio de Frías (2016) evidencia que los principales perpetradores de mujeres adultas mayores son sus hijos e hijas adultos. Como se muestra en la tabla 7, la violencia proveniente de estas personas también es una cuestión de género. Mientras los hijos son acusados de descuidar financieramente a sus madres, de gritarles y pegarles y hacerlas sentir como una carga económica, las mujeres son acusadas de negarles cuidados y de hacerlas sentir como una carga. Por lo tanto, es posible que las hijas cometan más violencia al quedarse en casa y estar en contacto frecuente con sus madres.

Asimismo, dos investigaciones señalaron que las mujeres adultas mayores expuestas a violencia física presentaron un mayor riesgo de letalidad como consecuencia de esos incidentes (Andrade et al., 2016; Curro et al., 2017). La tasa de letalidad crece con la edad: desde 0,7 para mujeres de 20 a 29 años hasta 3,5 para mujeres de ochenta años a más, como puede verse en la tabla 8 , tomada de un estudio conducido en Minas Gerais en Brasil por Andrade et al. (2016).

Tabla 6. Relación entre la edad de la víctima y el tipo de perpetrador, Brasil 2010

\begin{tabular}{lccccc}
\hline \multirow{2}{*}{ Grupo etario } & \multicolumn{5}{c}{ Perpetrador (\%) } \\
& Pareja & Familiar & Conocido & Desconocido & Otros \\
\hline Niñas y adolescentes & 25,2 & 24,9 & 21,1 & 16,1 & 12,7 \\
Mujeres adultas & 50,8 & 17,1 & 12,1 & 11,1 & 8,9 \\
Mujeres adultas mayores & 12,9 & 52,1 & 15,0 & 6,4 & 13,6 \\
Total & 45,2 & 19,2 & 13,6 & 11,7 & 9,7 \\
\hline
\end{tabular}

Fuente: Datos de Leite et al. (2014, p. 89). 
Tabla 7. Tipo de violencia experimentada por mujeres adultas mayores mexicanas según su perpetrador, México, 2011

\begin{tabular}{lcc}
\hline \multicolumn{1}{c}{ Tipos de violencia } & Hijo & Hija \\
\hline Física & & \\
Herir, golpear o lanzar cosas & 18,87 & 31,14 \\
Psicológica & & \\
Gritar, insultar u ofender & 29,28 & 31,14 \\
Ignorarla & 26,91 & 23,57 \\
Hacerla sentir o decirle que es una carga & 39,32 & 33,30 \\
Amenazarla con abandonarla en la calle & 20,80 & 32,27 \\
Negligencia & & \\
No cuidarla cuando está enferma, no & 53,69 & 49,60 \\
comprarle medicinas & & \\
Dejarla sola o abandonarla & 45,99 & 51,99 \\
Forzarla a realizar tareas domésticas & 36,16 & 43,52 \\
Negarle ayuda & 49,14 & 47,33 \\
Dejarle de dar dinero & 44,31 & 65,14 \\
\hline
\end{tabular}

Fuente: Frías (2016, p. 357). Elaboración y traducción propias.

Tabla 8. Distribución de la tasa de letalidad por VCM según grupo etario, Brasil, 2012

\begin{tabular}{cc}
\hline Grupo de edad & Tasa de letalidad (\%) \\
\hline $20-29$ & 0,7 \\
$30-39$ & 1,0 \\
$40-49$ & 1,4 \\
$50-59$ & 1,7 \\
$60-69$ & 2,1 \\
$70-79$ & 1,6 \\
$80+$ & 3,5 \\
\hline
\end{tabular}

Fuente: Datos de Andrade et al. (2016, p. 8). 
Los estudios cualitativos aportaron importantes conocimientos al describir los mundos sociales y las experiencias de violencia de mujeres adultas mayores a lo largo de la vida. Señalan que estas generaciones de mujeres fueron socializadas en contextos que legitimaron la desigualdad de género y permitieron la VCM. Estos sistemas de creencias fueron transmitidos a través de diferentes instituciones, como la religión o la familia, en contextos urbanos y rurales, y fueron incorporados por las mujeres participantes de estos estudios. Por ejemplo, López y Carrasco plantearon que las mujeres fueron educadas para seguir lo que llamaron «la norma del silencio» (2013, p. 40) y que construyeron sus identidades femeninas en torno a los valores de sumisión, perdón y la obediencia a la pareja y la responsabilidad de cuidar a otros miembros de sus familias, asumiendo el rol de madres y esposas.

Luego de incorporar estas ideas, las mujeres entrevistadas las reprodujeron en sus dinámicas familiares y de pareja. Por ejemplo, el estudio de Sepúlveda (2016), que analizó las historias de vida de veintiún mujeres adultas mayores católicas en Chile, explica que la mayoría de las mujeres de su generación (97\%) se socializaron alrededor de estas creencias y tradiciones religiosas. Esto supone una fuerte influencia en sus percepciones y experiencias sobre el matrimonio y la maternidad. Todos estos estudios sugieren que las mujeres adultas mayores con frecuencia normalizan la violencia que experimentan como algo que forma parte de la condición de ser mujer, mientras que algunas sí perciben estas situaciones como abuso, pero se sienten incapaces de cambiarlas o escapar de las mismas. De igual modo, Orbea (2017), que condujo entrevistas a profundidad a veintiún mujeres adultas mayores cubanas para evidenciar la normalización de la violencia de pareja, mostró que la mayoría de las víctimas entrevistadas consideraban que su relación marital era buena, mientras que solo un pequeño grupo las valoró como regulares o malas.

Este sistema de creencias sobre los roles y comportamientos de género es considerado una barrera al denunciar la violencia ejercida por la pareja. Orbea (2017) y Sepúlveda (2016) sugieren que las mujeres adultas mayores entrevistadas en Cuba y Chile no terminaron sus relaciones porque entendían al matrimonio como un lazo sagrado que solo podía romperse con la muerte y que debía mantenerse a todo costo. Además, Sepúlveda identificó otros valores católicos que dificultaban o retrasaban la búsqueda de ayuda. Algunas mujeres mantuvieron sus experiencias de violencia ocultas, pues consideraban a Dios como único confidente. Asimismo, las mujeres estaban socializadas en los valores de sumisión, resignación, sacrificio y perdón, lo que las hacía dejar a un lado sus 
propias necesidades y permanecer en una relación violenta, buscando garantizar la estabilidad económica de sus hijos. De igual forma, en la vejez, ellas se sacrifican a sí mismas por hacerse cargo del cuidado de sus parejas mayores. Estas creencias son retratadas en el siguiente testimonio, tomado de la investigación de Sepúlveda:

Cuando estaba muy triste [como resultado de estar en un matrimonio abusivo] nosotras rezábamos juntas [con una monja], eso me hacía sentir mucho mejor [...] Ella me dijo que seguramente era su tipo de personalidad. Que si él [el abusador] seguía casado conmigo era porque él debía de seguirme amando. Ella dijo que los lazos del Señor no deberían romperse a pesar de todas las dificultades [...], que cada uno de nosotros carga una cruz. Esa era mi cruz, y yo tenía que cargarla como símbolo de amor por Dios. Yo lo entendí de esa forma. Esa era mi cruz (Sepúlveda, 2016, p. 339).

Estos estudios retratan los dilemas que las mujeres adultas mayores enfrentan al decidir si denunciar a miembros de su familia como abusivos. Por ejemplo, el siguiente testimonio tomado de la investigación realizada por Souto et al. (2015) en Brasil muestra que las víctimas frecuentemente niegan u ocultan incidentes de violencia para proteger a sus perpetradores. Que las mujeres adultas mayores sean socializadas como cuidadoras y que mantengan sentimientos afectivos hacia sus perpetradores se convierten en barreras para la denuncia. Sus mayores expectativas y deseos son los de cambiar el comportamiento del agresor.

Mis otros hijos están molestos. Tú lo escuchaste diciendo que yo niego cosas que pasan en casa, y es cierto. Yo lo niego porque no quiero una pelea entre mis hijos. Así que lo escondo. Lo escondo para no empeorar la situación. Para evitar el conflicto, yo llamo y pido un carro para que me lleve a casa de mi otro hijo o a la granja, pero siempre dejo comida, carne en la refrigeradora, dejo todo para no dejarlo con hambre (Souto et al., 2015, p. 5).

Adicionalmente, el estudio de Hirt et al. (2018) en una comunidad rural en Brasil indicó que además existen barreras estructurales en la denuncia de la violencia, por ejemplo, «la ausencia de recursos colectivos de cuidado y protección sociales en contextos rurales, junto con la distancia geográfica hasta los centros urbanos» (Hirt et al., 2018, p. 2).

Acerca de las redes comunitarias de apoyo, los estudios cuantitativos indican que su presencia disminuye el riesgo de violencia. Por ejemplo, Rodriguez y Esquivel (2020) sugieren que la probabilidad de experimentar abuso disminuyó en un $10 \%$ para mujeres adultas mayores mexicanas que contaban con redes 
de apoyo. Al mismo tiempo, los estudios cualitativos explican que la violencia experimentada durante el curso de vida debilita las redes de apoyo familiar y comunitario de las mujeres adultas mayores en general. Sin embargo, algunas mujeres adultas mayores consiguieron mantener fuertes relaciones con algunos miembros clave de su familia que las protegen cuando necesitan ayuda (Orbea, 2017). Otras han logrado crear nuevas redes dentro de sus comunidades en forma clandestina, demostrando su agencia. Esto último está ilustrado en el siguiente testimonio, tomado del estudio realizado por Bruno y Castro (2019). Los investigadores analizaron el caso de Reyna, una mujer mexicana de 68 años que sufría de violencia de pareja. Secretamente, ella participaba en un Centro de Día para el Adulto Mayor buscando un nuevo espacio de socialización. Este servicio también le proporcionó apoyo instrumental — proveyéndola con algunos servicios esenciales de salud y necesidades materiales- y el apoyo emocional que no podía encontrar en su familia.

Usualmente vengo [al Centro de Día para el Adulto Mayor], y [mi esposo] no lo sabe. Eso lo enojaría, y yo no quiero quedarme atrapada en mi casa. Lloro mucho por cualquier cosa. Estoy siempre preocupada. Así que he decidido venir aquí, me invitaron. Durante el día, durante este momento, me siento aliviada, me siento como una persona diferente. No estoy en casa donde sólo pienso y pienso y lloro y lloro. Mis hijos sí saben, pero él no sabe que yo vengo aquí, yo vengo sin su permiso (Bruno y Castro, 2019, p. 77).

Finalmente, la investigación conducida por Fernandez y Garcia (2010) en Brasil mostró las consecuencias de la violencia en los cuerpos de las mujeres adultas mayores. Sus cuerpos y experiencias de vejez estaban marcados por el impacto de la desigualdad de género durante el transcurso de su vida, así como también por sus experiencias de maternidad y el abuso perpetrado por sus parejas durante décadas. Las participantes de este estudio consideraron que sus cuerpos habían envejecido prematuramente y se han enfermado a causa de estas situaciones. Ellas perciben que esos episodios de violencia y su sufrimiento se han acumulado y materializado en sus cuerpos envejecidos, dejando en ellas una marca permanente.

\section{DISCUSIÓN Y CONCLUSIONES}

Esta revisión crítica de la literatura ha identificado que el estudio de la violencia contra mujeres adultas mayores ha transcurrido por caminos similares en ALC, 
Europa y Estados Unidos. Tal como Hightower (2010) describió, el estudio de esta problemática social ha sido dominado por los marcos conceptuales de MPAM y VCM, y en menor medida por el de la interseccionalidad. A través de esta revisión, encontré que los primeros dos han dominado el estudio de este tema empleando un enfoque médico y metodologías cuantitativas, como en las investigaciones iniciales en países desarrollados.

La contribución más significativa de este grupo de estudios ha sido el hecho de poner en evidencia que las mujeres adultas mayores también experimentan violencia en la vejez. Sin embargo, su limitación más importante fue la incapacidad de analizar esta cuestión como un problema basado tanto en el género como en la edad. Así, pues, estos marcos abordaron la violencia de manera fragmentada, enfocándose sólo en una de estas características (edad o género) o solo en una etapa de la vida de las mujeres adultas mayores, como han criticado Band-Winterstein y Eisikovits (2009) y Hightower (2010) anteriormente. En distinción de lo encontrado en el Hemisferio Norte (Vinton, 1991; Fisher et al., 2003; Bows, 2019), los estudios sobre el MPAM en ALC han mostrado más interés que los estudios de VCM por explicar las causas de la violencia que las mujeres adultas mayores experimentan. Por tanto, como apuntaron los académicos brasileños Motta (2010) y Hightower (2010), la academia feminista ha mostrado más indiferencia hacia este tema que los académicos que trabajan desde el marco teórico del MPAM, lo que indica la existencia de una brecha de conocimiento y viejismo dentro de este movimiento social de gran trayectoria en ALC.

En comparación, un novedoso grupo de estudios realizados por investigadores sociales a través del marco conceptual de la interseccionalidad ha probado su potencial para resolver los problemas presentes en los otros campos de estudio, permitiéndonos comprender la experiencia de violencia de las mujeres adultas mayores integralmente. La contribución de los estudios cuantitativos interseccionales consistió en demostrar la importancia de enfocar la violencia desde una perspectiva de curso de vida para revelar su naturaleza acumulativa y la interconexión entre las diferentes experiencias de abuso a lo largo de la vida (Frías, 2016; Rodriguez y Esquivel, 2020). Sin embargo, como ya señalaron anteriormente la Cepal (2014) y González (2014), su principal limitación fue la dificultad para reconocer la verdadera magnitud de la violencia contra las mujeres adultas mayores debido a la escasez de datos estadísticos representativos sobre este tema en la mayoría de los países de ALC.

Los estudios cualitativos interseccionales demostraron que la aplicación de un enfoque fenomenológico permitía explorar otros aspectos de la violencia contra 
las mujeres adultas mayores nunca antes estudiados en la región. Por ejemplo, esos estudios exploraron los sistemas de creencias de las mujeres adultas mayores, sus experiencias cotidianas y percepciones de violencia, sus dilemas y barreras para buscar ayuda (Souto et al., 2015; Sepúlveda, 2016; Orbea, 2017). Uno de los aspectos clave que quisiera resaltar acerca de este grupo es que no solo retratan a las mujeres adultas mayores como víctimas, sino como sobrevivientes con agencia, describiendo cómo ellas utilizan diversas redes de soporte para sobrellevar situaciones violentas (Orbea, 2017; Bruno y Castro, 2019; Rodríguez y Esquivel, 2020).

Si bien este grupo de estudios aún es escaso en la región, esta tesis plantea que poseen un gran potencial para la comprensión de este novedoso tema a través de las perspectivas, los mundos sociales y las experiencias cotidianas de las víctimas. La inclusión de las percepciones y experiencias de mujeres adultas mayores en la construcción del conocimiento científico fue un reclamo de académicos estadounidenses, europeos (Hightower, 2010; Band-Winterstein y Eisikovits, 2010; Daichman, 2005) y latinoamericanos (Cartagena y Curcio, 2019). Ello permite la construcción de teoría de forma inductiva y basada en evidencia contextualizada es un arma poderosa para enfrentar falencias de los marcos teóricos anteriores que reproducen ideas viejistas y sexistas presentes en la academia gerontológica y feminista. Por último, es crucial crear marcos interpretativos adaptados a nuestros contextos culturales.

Finalmente, se brindarán algunas recomendaciones a los investigadores y formuladores de políticas de América Latina para alentar y orientar estudios futuros sobre violencia contra las mujeres adultas mayores, así como para contribuir en el diseño y mayor desarrollo de políticas públicas y servicios regionales y nacionales adaptados a las características y necesidades de este grupo de mujeres en nuestros países.

Por un lado, en ALC deberían realizarse nuevas investigaciones cualitativas y cuantitativas centradas en la violencia contra las mujeres de edad, pues se trata de una brecha de conocimiento en la mayoría de los países de la región que debería ser abordada en el futuro. Es crucial realizar esos estudios en los países más envejecidos de la región (Uruguay, Cuba, Chile, Argentina, Trinidad y Tobago) y en los que tienen tasas más altas de VCM (Bolivia, Ecuador, Costa Rica, Colombia, Trinidad y Tobago, Perú). Además, como ha sido sugerido anteriormente por investigadores y organismos internacionales (Daichman y Giraldo, 2013; OMS, 2014; Bott et al., 2012; Cepal, 2014), los estudios cuantitativos en ALC deberían analizar encuestas disponibles que incluyan información relacionada con este 
problema. Por ejemplo, las encuestas que se centran en la VCM y que no tienen límites de edad - recientemente creadas en el Ecuador, México, el Uruguay y el Perú - y las encuestas sobre el MPAM disponibles en México, el Ecuador y Colombia. Asimismo, la investigación cualitativa debería ser una prioridad para el desarrollo de interpretaciones teóricas de la violencia contra las mujeres adultas mayores en los países de América Latina y el Caribe.

Por otro lado, es necesario que los gobiernos de los países de ALC financien encuestas representativas sobre la violencia contra las mujeres adultas mayores para contar con información confiable sobre su prevalencia y magnitud. Estos datos podrían obtenerse eliminando los límites de edad de las encuestas demográficas y de salud reproductiva - DHS y RHS - o diseñando y realizando encuestas sobre el MPAM que permitan comparaciones de género. Los cuestionarios de estas encuestas deberían permitir cruces comparativos entre países homogenizando sus preguntas y operacionalización de sus variables de estudio, ya que, como Daichman y Giraldo (2013) y Gonzalez (2014) han enfatizado anteriormente, la falta de homogeneidad de la definición de violencia en estudios anteriores hace que los hallazgos sean imposibles de comparar o sistematizar. Además, la información recopilada por encuestas nacionales e investigaciones académicas debe utilizarse para la creación de servicios públicos basados en evidencia para la protección de las mujeres adultas mayores víctimas de violencia. Estos servicios deben tener como objetivo atender las necesidades particulares de este grupo de mujeres y formular estrategias para superar las barreras con las que lidian en la búsqueda de ayuda.

\section{REFERENCIAS}

Abath, M., Leal, M. y Melo, D. (2012). Factors associated with physical domestic violence in elderly victims. Revista Brasileira de Geriatria e Gerontologia, 15, 305-314. https://doi.org/10.1590/S1809-98232012000200013

Abath, M.B, Leal, M.C.C., Melo, D.A., Marques, A.P.O. (2010). Physical abuse of older people reported at the Institute of Forensic Medicine in Recife, Pernambuco State, Brazil. Cadernos de Saúde Pública, 26(9), 1797-1806. https://doi. org/10.1590/S0102-311X2010000900013

Agudelo, M. C., Cardona, D., Segura, A., Segura, A., Muñoz Rodríguez, D. y Restrepo, D. A. (2019). Social and family characteristics associated with elder abuse in Pasto, Colombia 2016. CES Psicología, 12(1), 32-42. https://doi.org/10.21615/ cesp.12.1.3 
Alarcon, M.F.S., Damaceno, D.G., Lazarini, C.A., Braccialli, L.A.D., Sponchiado, V.B.Y y Marin, M.J.S. (2019). Violence against the elderly: A documentary study. Rev Rene, 20, 1-9. https://doi.org/10.15253/2175-6783.20192041450

Alencar, F. y Moraes, J. (2018). Prevalence and factors associated with violence against elderly committed by strangers, Brazil, 2013. Epidemiologia e Serviços de Saúde, 27. https://doi.org/10.5123/S1679-49742018000200009

Andrade, J. O., Castro, S. S.C., Heitor, S.F.D., Andrade, W.P. y Atihe, C. C. (2016). Indicators of violence against women according to the reports of health services in the state of Minais Gerais-Brazil, Texto \& Contexto - Enfermagem, 25(03), e2880015-e2880015. https://doi.org/10.1590/0104-07072016002880015

Avanci, J., Pinto, L. y Assis, S. (2017). Treatment for cases of violence by Brazilian emergency services focusing on family relationships and life cycles. Ciencia \& Saúde Coletiva, 22, 2825-2840. https://doi.org/10.1590/1413-81232017229.13352017

Azevedo, E. y Tavares, M. (2014). As deams desconhecem sexo e gênero na velhice: Reflexões sobre mais uma modalidade de violência contra as mulheres. $18^{\circ}$ REDOR. Brazil.

Band-Winterstein, T. y Eisikovits, Z. (2009). Aging out of violence: The multiple faces of intimate violence over the life span. Qualitative Health Research, 19(2), 164-180. https://doi.org/10.1177/1049732308329305

Band-Winterstein, T. y Eisikovits, Z. (2010). Towards phenomenological theorizing about old women abuse. Ageing International, 35(3), 202-214. https://doi. org/10.1007/s12126-010-9067-y

Bardales, O. y Menéndez, B. (2015). Violencia familiar y sexual en las personas adultas mayores y su demanda a los servicios de atención a la violencia familiar y sexual. Perú: MIMP.

Batista, C. y Motta, A. (2014). Velhice é uma ausência? uma aproximação aos feminismos e à perspectiva geracional. Feminismos, 2(1967), 37-46.

Bernardino, I. M., Barbosa, K. G. N., Nóbrega, L.M., Cavalcante, G. M.S., Ferreira e Ferreira, E. y d'Avila, S. (2016). Violence against women in different stages of the life cycle in Brazil: An exploratory study. Revista Brasileira de Epidemiologia, 19(4), 740-752. https://doi.org/10.1590/1980-5497201600040005

Bolsoni, C.C., Lindner, S.R., D’Orsi, E. y Coelho, E.B.S. (2019). Dimensions of violence against the elderly and health conditions in southern Brazil. RaspResearch on Ageing and Social Policy, 7(2), 403-426. https://doi.org/10.17583/ rasp.2019.4425

Bonomi, A.E., Anderson, M.L., Reid, R. J., Carrell, D., Fishman, P.A., Rivara, F.P. y Thompson, R. S. (2007). Intimate partner violence in older women. The Gerontologist, 47(1), 34-41. https://doi.org/10.1093/geront/47.1.34 
Bott, S., Guedes, A., Goodwin, A. y Adams-Mendoza, J. (2012). Violencia contra las mujeres en América Latina y el Caribe. Análisis comparativo de datos poblacionales de 12 países. Washington DC: OMS, OPS.

Bows, H. (2019). Violence against older women. Volume I: Nature and Extent. UK: Palgrave. https://doi.org/10.1007/978-3-030-16601-4_1

Brandl, B. (2006). Understanding elder abuse. En B. Brandl et al. (eds.), Elder abuse detection and intervention: A collaborative approach (pp. 3-57). USA: Springer.

Brownell, P. (2016). A reflection on gender issues in elder abuse research: Brazil and Portugal. Ciencia \& Saúde Coletiva, 21,3323-3330. https://doi.org/10.1590/1413812320152111.23142016

Brownell, P. (2019). Future directions for research on neglect, abuse and violence against older women. South Eastern European Journal of Public Health, 11(February), 1-8.

Bruno, F. y Castro L. K. (2019). Violencia hacia las mujeres adultas mayores y redes de apoyo social en Monterrey, México. Un aporte desde el construccionismo social. Perspectivas sociales, 21, 55-85.

Calasanti, T. y King, N. (2015). Intersectionality and Age. En J. Twigg y W. Martin (eds.), Routledge Handbook of Cultural Gerontology (pp. 193-201). Nueva York: Routledge.

Calasanti, T. y Slevin, K. (2006). Age matters. Realigning feminist thinking. Nueva York y Londres: Routledge.

Cartagena, E. A. y Curcio, C. L. (2019). Elder abuse in the city of Manizales (Colombia): A gender perspective. Revista Latinoamericana de Estudios de Familia, 11, 111128.

Castro, V., Rissardo, L. y Carreira, L. (2018). Violence against the Brazilian elderlies: An analysis of hospitalizations. Revista Brasileira de Enfermagem, 71, 777-785. https://doi.org/10.1590/0034-7167-2017-0139

Cotlear, D. y Tornarolli, L. (2011). Pobreza, adultos mayores y el ciclo de vida en América Latina. En D. Cotlear (ed.), Envejecimiento de la población: ¿está preparada América Latina? (pp. 79-134). USA: World Bank.

Crenshaw, K. (1989). Demarginalizing the intersection of race and sex: A black feminist critique of anti-discrimination doctrine, feminist theory and antiracist politics. University of Chicago Legal Forum, 1, 139-168.

Crockett, C. y Cooper, B. (2016). Gender norms as health harms: Reclaiming a life course perspective on sexual and reproductive health and rights. Reproductive Health Matters, 24(48), 6-13. https://doi.org/10.1016/j.rhm.2016.11.003 
Crockett, C., Brandl, B. y Dabby, F. C. (2015). Survivors in the margins: The invisibility of violence against older women. Journal of Elder Abuse \& Neglect, 27(4-5), 291-302. https://doi.org/10.1080/08946566.2015.1090361

Curcio, C., Payan, C., Jimenez, A. y Gómez, F. (2019). Abuse in Colombian elderly and its association with socioeconomic conditions and functionality. Colombia Médica, 50(2), 77-88. https://doi.org/10.25100/cm.v50i2.4013

Curro Urbano, O. M., Pastor Ramírez, N., Hernández Huaripaucar, E. M., Chauca Saavedra, C. L., Puza Mendoza, G. M., Córdova Delgado, M., Quispe Ilanzo, M. P., y Oyola García, A.E. (2017). Violencia extrema contra la mujer y feminicidio: del escenario íntimo al tráfico de personas en el Perú. Cuadernos de Medicina Forense, 23(1-2), 15-23.

Daichman, L. (2005). Elder abuse in developing nations. En M. Johnson et al. (eds.), The Cambridge handbook of age and ageing (pp. 323-332). Cambridge: Cambridge University Press. https://doi.org/10.1017/CBO9780511610714.032

Daichman, L. y Giraldo, L. (2013). Latin America. En A. Phelan (ed.), International Perspectives on Elder Abuse (pp. 134-150). Oxon y Nueva York: Routledge.

Díaz, C., Ellertson, C., Paz, F., Ponce de León, S. y Alarcon, D. (2002). Prevalence of battering among 1780 outpatients at an internal medicine institution in Mexico. Social Science \& Medicine, 55(9), 1589-1602. https://doi.org/10.1016/S02779536(01)00293-3

Dulcey-Ruiz, E. (2013). Envejecimiento y vejez. Categorías conceptuales. Bogotá: Red Latinoamericana de Gerontología and Fundación Cepsiger para el Desarrollo Humano.

ECLAC (2014). Annual Report 2013-2014. Confronting violence against women in Latin America and the Caribbean. Santiago de Chile: ECLAC.

ECLAC (2016). The social inequality matrix in Latin America. Santiago de Chile: ECLAC.

Enamorado Pina, G. V., Perez Rodríguez, J., Domínguez Cancino, A. y Rodriguez Enamorado, J.E. (2013). Violencia intrafamiliar contra el adulto mayor en una comunidad de Guinea Bissau. MEDISAN, 17(7), 1053-1059.

Faustino A., Moura, L. y Gandolfi, L. (2014). Sociodemographic profiles and violent events experienced by the elderly: A descriptive study. Online Brazilian Journal of Nursing, 13, 529-536. https://doi.org/10.5935/1676-4285.20144588

Fernandes, M. y Garcia, L. (2010). The aged body: Perception and experience of elderly women. Interface: Communication, Health, Education, 14(35), 879-890. https:// doi.org/10.1590/S1414-32832010005000024 
Fisher, B. S., Zink, T. M., Rinto, B. A., Regan, S. L., Pabst, S. R. y Gothelf, E. J. (2003). Overlooked issues during the golden years: domestic violence and intimate partner violence against older women. Violence Against Women, 9(12), 1409-1416. https://doi.org/10.1177/1077801203259230

Frías, S. (2016). Polivictimización en mujeres mexicanas adultas mayores. Revista Mexicana de Sociología, 78, 343-374.

Gaioli, C. y Rodrigues, R. (2008). Occurrence of domestic elder abuse. Revista Latino-Americana de Enfermagem, 16(3), 465-470. https://doi.org/10.1590/ S0104-11692008000300021

Garbin, C.A. S., Joaquim, R. C., Rovida, T.A. S., Garbin, A. J. I. (2016). Elderly victims of abuse: a five year document analysis. Revista Brasileira de Geriatria e Gerontologia, 19(1), 87-94. https://doi.org/10.1590/1809-9823.2016.15037

Garcia, L., Duarte, E., Freitas, L., Silva, G. D. M. (2016). Domestic and family violence against women: a case-control study with victims treated in emergency rooms. Cadernos de Saúde Pública, 32(4). https://doi.org/10.1590/0102-311X00011415

Giraldo, L. (2010). El maltrato a personas adultas mayores: una mirada desde la perspectiva de género. Debate feminista, 42, 151-165. https://doi.org/10.22201/ cieg.2594066xe.2010.42.825

González, K. (2014). Análisis de los factores asociados a la violencia contra mujeres de 60 años y más en México. Social Sciences. Mexico: FLACSO.

Guedes, D. T., Alvarado, B. E., Phillips, S. P., Curcio, C. L., Zunzunegui, M. V., Guerra, R.O. (2015). Socioeconomic status, social relations and domestic violence (DV) against elderly people in Canada, Albania, Colombia and Brazil. Archives of Gerontology and Geriatrics, 60(3), 492-500. https://doi.org/10.1016/j.archger.2015.01.010

Guedes, D. T., Curcio, C.L., Alvarado, B.E., Zunzunegui, M. V., Guerra, R. O. (2015). The gender gap in domestic violence in older adults in Latin America: the IMIAS Study. Pan American Journal of Public Health, 37(4-5), 293-300.

Hawker, S., Payne, S., Kerr, C., Hardey, M. y Powell, J. (2002). Appraising the evidence: Reviewing disparate data systematically. Qualitative Health Research, 12(9), 1284-1299. https://doi.org/10.1177/1049732302238251

HelpAge International (2017a). How data systems leave older people behind. Londres: Economic \& Social Research Council.

HelpAge International (2017b). Violence against older women. Londres: Help Age International.

Hightower, J. (2004). Age, gender and violence: abuse against older women. Women's Health, 7(3), 60-63. 
Hightower, J. (2010). Abuse in later life: when and how does gender matter? En G. Gutman y C. Spencer (eds.), Aging, Ageism and Abuse. Moving from awareness to action (pp. 17-30). Londres: Elsevier. https://doi.org/10.1016/B978-0-12381508-8.00002-3

Hirt, M. C., Costa, M. C.D., Arboit, J., Leite, M. T., Hester, L.Z., Silva, E. B. D. (2018). Social representations of violence against women for a group of rural elderly. Revista Gaúcha de Enfermagem, 38, e68209-e68209.

Huenchuan, S. (2018). Envejecimiento, personas mayores y Agenda 2030 para el Desarrollo Sostenible: perspectiva regional y de derechos humanos. Santiago de Chile: ECLAC. https://doi.org/10.18356/19532890-es

IDB (2015). Pensions at a Glance: Latin America and the Caribbean. USA: IDB.

Jiménez, A. (2012). Violencia en la vejez: el caso de las abuelas que cuidan a nietos y nietas en una localidad rural en el estado de Hidalgo. El Cotidiano, 174, 19-32.

Kalache, A., Barreto, S. y Keller, I. (2005). Global ageing: The demographic revolution in all cultures and societies. En M. Johnson (ed.), The Cambridge Handbook of Age and Ageing (pp. 30-46). Cambridge: Cambridge University Press. https:// doi.org/10.1017/CBO9780511610714.005

Leeson, G. (2013). The demographics of population ageing in Latin America, and the Caribbean and the Iberian Peninsula, 1950-2050. En V. Montes de Oca (ed.), Envejecimiento en América Latina y el Caribe. Enfoques en investigación y docencia de la Red Latinoamericana de Investigación en Envejecimiento (LARNA) (pp. 53-74). México: UNAM.

Leite, M.T.S., Figueiredo, M.F.S., Dias, O.V., Vieira, M.A., Souza e Souza, L.P., Mendes, D.C. (2014). Reports of violence against women in different life cycles. Revista Latino-Americana de Enfermagem, 22(1), 85-92. https://doi. org/10.1590/0104-1169.3186.2388

López, C. y Carrasco, G. (2013). El silencio del trauma. Violencia acumulada en la vejez de las reinas del hogar. Anuac, 2, 30-50.

Manso, M.E. G. y Lopes, R. G.C. (2020). Violência contra a mulher idosa: estado da arte. Kairós-Gerontologia, 23(4), 65-80.

Markides, K., Salinas, J. y Wong, R. (2010). Ageing and health among Hispanics/ Latinos in the Americas. En D. Dannefer y C. Phillipson (eds.), The SAGE Handbook of Social Gerontology (pp. 150-163). Londres: SAGE. https://doi. org/10.4135/9781446200933.n11

Martina, M., Nolberto, V., Miljanovich, M., Bardales, O. y Gálvez, D. (2010). Violencia hacia el adulto mayor: Centros Emergencia Mujer del Ministerio de la Mujer y Desarrollo Social. Lima-Peru, 2009. Revista Peruana de Epidemiología, 14(3), 1-7. 
Mascarenhas, M.D.M., Andrade, S.S.C.A., Neves, A.C.M., Pedrosa, A.A. G., Silva, M. M.A., Malta, D.C. (2012). Violência contra a pessoa idosa: análise das notificações realizadas no setor saúde - Brasil, 2010. Ciência \& Saúde Coletiva, 17(9), 2331-2341. https://doi.org/10.1590/S1413-81232012000900014

McGarry, J. y Ali, P. (2019). The Invisibility of Older Women as Survivors of Intimate Partner Violence. En H. Bows (ed.), Violence against older women (pp. 41-56). Volume I: Nature and Extent. UK: Palgrave. https://doi.org/10.1007/978-3-03016601-4_3

Mears, J. (2003). Survival Is Not Enough: Violence Against Older Women in Australia. Violence Against Women, 9(12), 1478-1489. https://doi. org/10.1177/1077801203259287

Meirelles, R.C. Junior, Castro, J.O., Faria, L.R., Silva, C.L.A., Alves, W.A. (2019). Reporting of deaths from external causes and violence against older people: a veiled reality. Revista Brasileira em Promocão da Saúde, 32, 1-12. https://doi. org/10.5020/18061230.2019.8685

Meyer, S., Lasater, M. y Garcia-Moreno, C. (2019). Violence against older women: A protocol for a systematic review of qualitative literature. BMJ Open, 9(5), 1-6. https://doi.org/10.1136/bmjopen-2018-028809

Mikulionienė, S. y Tamutienė, I. (2019). Perceptions of Domestic Violence Against Older Women: When Sexism Is Compounded by Ageism. En H. Bows (ed.), Violence against older women (pp. 15-40). Volume I: Nature and Extent. UK: Palgrave. https://doi.org/10.1007/978-3-030-16601-4_2

Motta, A. (2010). A atualidade do conceito de gerações na pesquisa sobre o envelhecimento. Sociedade e Estado, 25(2), 225-250. https://doi.org/10.1590/ S0102-69922010000200005

Orbea, M. (2017). Until death us do part? Couple violence in the Third Age. Novedades en Población, 13, 134-144.

Patticrew, M. y Roberts, H. (2006). Systematic reviews in the social sciences: a practical guide. Oxford: Blackwell Publishing.

Ribot, V., Rousseaux, E., Garcia, T., Arteaga, E., Ramos, M. y Alfonso, M. (2015). Psychological the Most Common Elder Abuse in a Havana Neighborhood, Medicc Review, 17(2), 39-43. https://doi.org/10.37757/MR2015.V17.N2.9

Rodrigues, R. S. y Abadal, E. (2014). Ibero-American journals in Scopus and web of science, Learned Publishing, 27(1), 56-62. https://doi.org/10.1087/20140109

Rodríguez, R. y Esquivel, E. (2020). Prevalence and associated factors of intimate partner violence among elderly Mexican women. Salud Colectiva, 16, e2600. 
Ruelas, M., Duarte, M., Flores, S., Ortega, D., Cortés, J., Taboada, A. y Ruano, A. (2016). Prevalence and factors associated with violence and abuse of older adults in Mexico's 2012 National Health and Nutrition Survey. International Journal for Equity in Health, 15(35), 1-9. https://doi.org/10.1186/s12939-016-0315-y

Saad, P. (2011). Tendencias demográficas en América Latina y el Caribe. En D. Cotlear (ed.), Envejecimiento de la población: ¿está preparada América Latina? (pp. 45-78). USA: World Bank.

Sepúlveda, P. (2016). Religious beliefs and gender violence. Analysis of older women's life stories in Chile (1940-2010). Feminismo/s, 28, 315-344.

Souto, R.Q., Merighi, M.A.B., Guruge, S. y Jesus, M.C.P. (2015). Older Brazilian women's experience of psychological domestic violence: a social phenomenological study. International Journal for Equity in Health, 14(44), 1-9. https://doi. org/10.1186/s12939-015-0173-z

Straka, S. y Montminy, L. (2006). Responding to the needs of older women experiencing domestic violence. Violence against women, 12(3), 251-267. https://doi. org/10.1177/1077801206286221

United Nations (2013) Neglect, abuse and violence against older women. Nueva York: United Nations.

United Nations (2019). World Population Prospects 2019, custom data acquired via website.

United Nations (2020). World Population Ageing 2019. Nueva York: United Nations.

Vinton, L. (1991). Abused older women: Battered women or abused elders? Journal of Women \& Aging, 3(3), 5-19. https://doi.org/10.1300/J074v03n03_03

Weeks, L., Macquarrie, C., Begley, R., Gill, C. y Leblanc, K. D. (2016). Strengthening resources for midlife and older rural women who experience intimate partner violence. Journal of Women \& Aging, 28(1), 46-57. https://doi.org/10.1080/089 52841.2014 .950500

World Health Organization (2014). Global status report on violence prevention 2014. Ginebra: WHO.

World Health Organization e INPEA (2002). Missing voices. Views of older persons on elder abuse. Ginebra: INPEA; WHO.

Zink, T., Regan, S., Jacobson, C. J. y Pabst, S. (2003). Cohort, Period, and Aging Effects: A Qualitative Study of Older Women's Reasons for Remaining in Abusive Relationships. Violence Against Women, 9(12), 1429-1441. https://doi. org/10.1177/1077801203259231 